\title{
Spectral optimized asymmetric segmented phase-only correlation filter
}

\author{
I. Leonard", A. Alfalou, ${ }^{1}$ and C. Brosseau ${ }^{2}$ \\ ${ }^{1}$ ISEN Brest, Département Vision, L@bISEN, \\ 20 rue Cuirassé Bretagne, CS 42807, 29228 Brest Cedex 2, France \\ E-mail: ayman.al-falou@isen.fr \\ ${ }^{2}$ Université Européenne de Bretagne, Université de Brest, Lab-STICC, \\ CS 93837, 6 avenue Le Gorgeu, 29238 Brest Cedex 3, France \\ E-mail: brosseau@univ-brest.fr
}

OCIS codes: $070.0070,100.3008,100.5010,100.5090$. 


\begin{abstract}
We suggest a new type of optimized composite filter, i.e. the asymmetric segmented phase-only filter (ASPOF), for improving the effectiveness of a VanderLugt correlator (VLC) when used for face identification. Basically, it consists in merging several reference images after application of a specific spectral optimization method. After segmentation of the spectral filter plane to several areas, each area is assigned to a single winner reference according to a new optimized criterion. The point of the paper is to show that this method offers a significant performance improvement on standard composite filters for face identification. We first briefly revisit composite filters (adapted, phase-only, inverse, compromise optimal, segmented, minimum average correlation energy, optimal trade-off maximum average correlation, and amplitude modulated phase-only (AMPOF)) which are tools of choice for face recognition based on correlation techniques and compare their performances with those of the ASPOF. We illustrate some of the drawbacks of current filters for several binary and gray scale images identifications. Next, we describe the optimization steps and introduce the ASPOF that can overcome these technical issues to improve the quality and the reliability of the correlation based decision. We derive performance measures, i.e. peak-to-correlation energy values and receiver operating characteristic curves, to confirm consistency of the results. We numerically find that this filter increases the recognition rate and decrease the false alarm rate. The results show that the discrimination of the ASPOF is comparable to that of the AMPOF, but the ASPOF is more robust than the trade-off maximum average correlation height
\end{abstract}


$(\mathrm{OT}-\mathrm{MACH})$ against rotation and various types of noise sources. Our method has several features that make it amenable to experimental implementation using a VLC. 


\section{Introduction}

Intense interest in optical correlation techniques over a prolonged period has focused substantially on the filter designs. These techniques represent a powerful tool for a wide range of applications like target tracking, identification, and classification. These applications require a real time processing, must be robust to several perturbations, e.g. face rotation, and should be insensitive to noise. The optical correlation technique is a good candidate for very fast recognition systems. However, the principle of correlation based on a comparison between the target image and a correlation filter makes this method not robust to rotation. One of the techniques used to overcome this problem is the composite filter, i.e. it contains information coming from multiple images. Based on the composite technique, the overall goal of this line of research is to develop and optimize a novel filter for the VanderLugt correlator used in face recognition [1] that is able to fulfill the above requirements and can handle a large database. In this study, we use different faces with several viewpoints from the Pointing Head Pose Image Database (PHPID) [2]. The results will show the impact of using the fusion criterion permitting to assign a single reference to each filter-pixel. Moreover, thanks to the symmetry property of the spectrum the number of real references in the composite filter is increased. This has for effect to improve significantly the decision making process.

Following this brief introduction, we have divided the rest of the paper as follows: a general overview of optical correlation methods is given in Sec. 2 providing technical details for the system under consideration, and a connection to earlier ideas. In Sec. 3, we present a number of relevant examples to illustrate our strategy for improving the effectiveness of the VLC using the asymmetric segmented phase-only filter (ASPOF). This face recognition algorithm was compared to a whole set of composite correlation filters: all systems were trained and tested on 
the same images. Our conclusion is that the increase of performance obtained with the ASPOF indicates that we were able to propose and validate a new composite correlation filter allowing us to increase considerably the number of references to be incorporated in one filter. The ROC curves permitted to distinguish between the good recognition and the true non recognition values based on an optimized PCE criterion. Finally, we draw our conclusions in Sec. 4.

\section{Some preliminary considerations and relation to previous work}

The optical correlation method can be traced back to the pioneering research in the $1960 \mathrm{~s}[1,3]$. This method consists of comparing a target image and a reference image that comes from a database. This comparison leads to correlation peak in the correlation plane. This correlation peak is more or less intense, depending on the degree of resemblance between the target and reference images. To optimize recognition, several filters created from the reference image have been studied [4-6]. However, the correlation approach which needs to compare the target image with a large number of reference images in order to increase its robustness is time consuming. To reduce the processing time, several composite filters have been developed [6-11].

Over the last decade, there has been a resurgence of interest, driven by recognition and identification applications [12-17], of the correlation methods. For example, Alam et al. [17] demonstrated the good performances of the correlation method compared to all numerical ones based on the independent component model. Another significant example in this area of research is the work by Romdhani et al. [18], which compared face recognition algorithms with respect to those based on correlation. These references are far from a complete list of important advances, but fortunately the interested reader can easily trace the historical evolution of these ideas with Vijaya Kumar's review paper, Yu's book, and the chapter of Alfalou and Brosseau containing an 
extensive bibliography $[4,6-7,19]$. According to the above considerations, our goal is to propose and validate a new robust and discriminating composite correlation filter which is able to include a large set of training images into this filter which allows one to deal with the diversity of facial expressions as input information. To proceed further, we begin with a brief overview of the VLC method. Next, we wish to specify the definition of standard performance measures that will be used to compare filters.

\subsection{VLC}

In this study we consider only the VLC scheme and we focus on the design of a new type of optimized composite filter. VLC was preferred to the joint transform correlator (JTC) [20-23] because it allows exploitation of all the finite bandwidth in the input plane [24]. Fig. (1) illustrates a simplified schematic of the VLC [1,4]. It is based on the multiplication between the spectrum, obtained by Fourier transforming the target image (Fig 2 (a)), with a correlation filter made from the FT of a reference image and placed in the Fourier plane. The correlation plane is obtained from $\mathrm{FT}^{-1}$ of the product of both spectra. This results in a more or less intense central correlation peak depending on the degree of similarity between the target object and the image reference. Many approaches for designing the correlation filter can be found in the literature according to the specific objects that need to be recognized [4, 6, 19, 25].

\subsection{Performance measures for filter design}

In order to quantify the correlation plane by the correlation of a filter and a test image different correlation plane performance measures were used [26-27]. We choose the PCE criterion [26-27] which is defined by 


$$
P C E=(\text { Energy in the correlation peak }) /(\text { overall energy in the correlation plane })
$$

In other words, the PCE must be interpreted as the weight of the output signal (correlation peak) in the correlation plane relative to noise. In practice, one should take into account of the width of the correlation peak in the calculation of the PCE. In what follows, PCE will be evaluated as

$$
\mathrm{PCE}=\sum_{x=x_{0}-t}^{x=x_{0}+t} \sum_{y=y_{0}-t}^{y=y_{0}+t}|C(x, y)|^{2} / \sum_{x=1}^{x=N} \sum_{y=1}^{y=M}|C(x, y)|^{2}
$$

where $\left(x_{0}, y_{0}\right)$ denote the position of the correlation peak, $C(x, y)$ denotes the value of the correlation plane at point $(x, y), t$ is set to the number of neighboring pixels used, and $(N, M)$ denote the size (in pixels) of the correlation plane. A distinct advantage of the PCE is that it permits evaluation of the relative importance of the correlation peak with reference to the noise of the correlation plane.

Among standard measures for parametrizing the correlator performances, the receiver operating characteristic (ROC) curve, which allow characterizing the binary classifiers, was selected [28]. The ROC curve can be represented by plotting the fraction of true positives out of the positives TPR (sensitivity, or true positive rate) versus the fraction of false positives out of the negatives FPR (1- specificity, or false positive rate). We find it can be more useful to consider adapted ROC curves [28]. We fix a weight according to the relative position of the PCE with respect to threshold. We shall consider 10 zones separating the threshold and the maximum PCE values obtained for a target face, and affect the weight $=0.1$ for the first zone, 0.2 to the second zone, and so on. 


\section{Asymmetric segmented composite POF (ASPOF): a spectral optimized filter}

The preceding analysis demonstrates the impact of a reduced number of correlations to obtain a reliable decision by using a composite filter. However, this filter needs to be optimized in order it can permits to merge a large number of reference images within a given filter. For that purpose we suggest a new composite filter, the ASPOF. The ASPOF is an optimized version of the segmented correlation filter [8]. Our algorithm has been extensively tested by comparing its performance with those of several composite filters [30].

\subsection{Segmented composite filter (SPOF)}

The main idea behind the SPOF is that the high saturation regions of the reference images are suppressed. Briefly stated, this is achieved through two steps [8]. First, a segmentation of the spectral plane of the correlation filter is realized into several independent regions. Second, each region is assigned to a single reference. This assignment is done according a specific energy criterion

$$
\frac{E_{u, v}^{l}}{\sum_{i, j} E_{i, j}^{l}}<\frac{E_{u, v}^{k}}{\sum_{i, j} E_{i, j}^{k}}
$$

where $E_{u, v}^{l}$ denotes the energy on the pixel $(u, v)$ of the reference image $(l)$ in the spectral domain. This criterion compares the energy (normalized by the total energy of the spectrum) for each frequency of a given reference with the corresponding energies of another reference. Assignment of a region to one of the two references is done according Eq. (3). Hence, the SPOF contains frequencies with the largest energy. 


\subsection{ASPOF algorithm}

A serious limitation of the SPOF is that the regions to which are assigned the winning references become smaller as the similarity between the reference images is increased. This effect eventually leads to isolated pixels. This issue can be critical since this paper deals with training sets of reference images bearing a strong resemblance, i.e. data-base obtained by rotating images within the range $\left(-90^{\circ},+90^{\circ}\right)$, and we want to increase the number of reference images in the filter. Our optimizing method follows from the four-step scheme which defines the ASPOF.

(i) We first separate the reference image base $\left(\left\{R_{i=1 \ldots N}\right\}\right.$, where $N$ denotes the number of used references), in two sub-classes: one, $\left\{R_{i}^{1}\right\}$, which contains the reference images with $i=1,3,5, \ldots, N-1$, the other one, $\left\{R_{i}^{2}\right\}$, deal with the other indices $i=2,4,6, \ldots, N$ (Fig. 3).

Next, in stage (ii), two segmented filters are constructed, using an analysis similar method to [29], for the two sub-classes, $H_{S P O F}^{1}$ and $H_{S P O F}^{2}$, respectively.

(iii) Selection and assignment optimized criterion used for segmentation of the Fourier plane for both filters are realized as follows

$$
H_{\text {SPOF }}^{1}=\left\{\begin{array}{c}
\text { Spect }_{u, v}^{l} \quad \text { if } \quad \frac{E_{u, v}^{l}}{\sum_{i, j} E_{i, j}^{l}} \geq a \frac{E_{u, v}^{k}}{\sum_{i, j} E_{i, j}^{k}} \\
\text { with } \quad l \in[0, N-1], \\
\text { Not affected } \quad \text { otherwise }
\end{array}\right.
$$

Here Spect ${ }_{u, v}^{l}$ is the spectrum of the reference $R_{l}$ at position $(u, v), E_{u, v}^{l}$ is the energy of the reference $R_{l}$ at the position $(u, v)$. The constant $a$, (here, set to 1.2), is a pixel assignment coefficient. Basically, it means that the pixel of the filter is assigned to a given reference if and only if its energy relative to spectral position $(u, v)$ is larger than the energies of all other 
references multiplied $a$ times. Otherwise, it is not assigned. In Eq. (4), the usefulness of the coefficient $a$ is motivated by the fact that sometimes there exist areas of the spectral plane which are very similar. Thus, by introducing this coefficient $a$ these areas are not assigned to any spectrum according to the segmentation criterion (Eq. 4). These areas will be assigned according the neighborhoods of each spectrum (Fig. 4). A similar operation for the second filter $H_{S P O F}^{2}$ is applied using the spectra of the second sub-class and considering again Eq. (4).

(iv) In this procedure, unclassified pixels, i.e. those which do not satisfy Eq. (4), are assigned to one of the two spectra by looking at their closest neighbors (Fig. 4) in order to avoid isolated pixels. An isolated pixel represents a pixel of a spectrum $l$ which is surrounded by pixels of the spectrum $k$. Isolated pixels are detrimental to the segmented filter's performance; the effect being more and more important as the number of references which define the filter is increased. Faced with several options for the purpose of decreasing this effect, we chose the following scenario. The assignment of pixels which do not satisfy Eq. (4) is realized according the schematic illustration depicted in Fig. 4. This figure show different situations, e.g. in Fig. 4 (a) and (b) the considered pixel is surrounded by pixels of only one spectrum.

Hence, the pixel is assigned to this spectrum. In another situation, Fig. 4 (c) illustrates the case of a pixel surrounded by pixels belonging to the two spectra. In this case, the algorithm searches for the immediate neighborhood ( $3 \times 3$ pixels) of the pixel, and the rule is to assign this pixel to the spectrum which has the largest number of pixels. Fig. 4 (d) represents an example in the case that the surrounding environment of the considered pixel contains exactly the same number of pixels for the two spectra. In this case, the algorithm searches for the spectrum that has the largest number of pixels in an enlarged neighbourhood, i.e. $5 \times 5$ pixels. 
(v) Next, the assignment step is repeated. Interestingly, we observed that a single iteration is necessary for letters.

(vi) Next, $H_{S P O F}^{1}$ is multiplied with a symmetric filter (denoted as filter 1 (1/0) in Fig. 5, and $H_{S P O F}^{2}$ is multiplied with the corresponding filter $2(0 / 1)$ in Fig. 5. Finally, we sum the two filters in the Fourier plane. Overall, the ASPOF can be described by $H_{A S P O F}=p_{1} H_{S P O F}^{1}$ filter $_{1}+p_{2} H_{S P O F}^{2}$ filter $_{2}$, with $p_{1}$ and $p_{2}$ being two coefficients. In the following we will study the effect of these two numbers on the performance of the ASPOF.

\subsection{ASPOF parameterization}

Two kinds of parameter were considered. On the one hand, to illustrate how $t$ in Eq. (2), i.e. $(2 t+1)^{2}$ pixels are required for the calculation of the PCE, can impact the correlation performances, we consider the letter A (with rotations between $-90^{\circ}$ and $90^{\circ}$ ) and compare the performances of the POF, SPOF, AMPOF, and ASPOF. The fabrication of these filters was realized using with 10 references, i.e. binary images of the letter A and 10 rotation angles. Fig. 6 compares the PCE resulting from correlating the letters A and V with the four composite filters. Taking $t=1$ pixel (Fig. 6 (a)), the ASPOF is found to be very discriminating. One way to increase the robustness of the ASPOF is to increase $t$. It can be seen that the ASPOF shows the best compromise between robustness and discrimination factor when $t=10$. For $t$ larger than 10 , the discrimination quality decreases (Fig. 6 (d)). In the following, $t$ is set to 10 . On the other hand, we further show how $p_{1}$ and $p_{2}$ can influence the performances of the ASPOF. Fig. 7 (a) shows the PCE values with $p_{1}=p_{2}=1$ where we choose the same illustrating example as above. We found by an iterative method (100 iterations, Fig 7 (b)) that the best performances were obtained by taking $p_{1}=2$ and $p_{2}=1.5$. 


\subsection{Composite filters used for comparison with the ASPOF}

In this subsection, we will review various composite filters in order to select some of them (POF, SPOF, AMPOF, and trade-off maximum average correlation height (OT-MACH)) that will be compared with the ASPOF in order to demonstrate its robustness and its good discrimination. We first study the effect of the background. We considered different classical composite filters (the full list is shown in Table 1). A detailed comparative study of the discrimination and robustness performances for standard composite correlation filters employed for face recognition is presented in the supplementary material [30].

As expected the adapted composite filter is robust against rotation and not discriminating. Moreover, its low discriminating character is more and more visible as the number of references is increased. Our findings suggest that the composite POF is quite robust but not much discriminating, see Fig. 8 and Fig. 9. For obtaining these figures we have correlated different rotations (from -90 to $90^{\circ}$ ) of the A and V letters with different composite POF filters using different rotations of the letter A as reference images. As seen in Fig. 8 the different PCE data show that the correlation values have an average PCE value larger than $310^{-3}$. It is important to point out that the PCE values obtained from the correlation of the letter V correlated with the same filters have an average value less than $10^{-3}$, and support the conclusions reported in [31]. In addition, composite POF show robustness to noise, especially when the noise is clearly identified. However, we find that the energy contained in the correlation peak decreases significantly, i.e. the PCE is decreased by a factor of 3 when using a POF containing 3 references by contrast with a POF realized with a single reference (Fig. 8). This supports also the conclusions made in [31]. Fig. (8) shows that for a 11-reference POF, the PCE is decreased by an 
order of magnitude which renders unreliable the decision on the letter identification. Beyond 11 references, the weakness of the magnitude of the PCE makes the recognition of the images forming the filter very difficult. This is partly due to the saturation. Indeed, when the number of training images is large saturation occurs because the correlation filter is pixelated, i.e. since ach pixel is encoded with a fixed number of bits, increasing the latter has for effect to slow down the filtering process and thus to increase the required memory space. To overcome this saturation limitation the segmented composite filter (SPOF) was proposed and validated in [8]. To further show the interest in using a segmented filter with respect to the saturation problem which affects the classical composite filter, we show in Fig. 2 (b) the 8-bit image of the sum (without segmentation) of the three spectra corresponding to the reference images (Fig. 2 (a)). Fig. 2 (c) shows the corresponding sum with segmentation [8]. Our calculations clearly indicate that the image with segmentation shows significantly less saturation than that obtained without segmentation.

For comparison, we performed simulations for a binarized composite POF [30]. Overall, the behavior of this kind of filter is very similar to that of POF albeit with smaller performances. We should notice that this conclusion is similar to that noted by Mohammed et al. in [32]. The low performances of the binarized POF is confirmed by a comparison between the results obtained by Mohammed et al. [32] and those reported by Rahman et al. [31].

Overall, we found that the composite POF has a good robustness against rotation but has a low discrimination. To look at the discrimination of composite filters in more detail, we shall consider the inverse composite filter. Such filter shows a strong discriminating ability and a low robustness against small changes of the target image with respect to the reference image [30]. Fig. 10 presents the correlation results obtained for different rotations of the letter A and several 
inverse filters. This leads to the conclusion that this inverse composite filter is not well adapted for the composite architecture.

Next, testing the compromise optimal filter, we observed that its composite version is robust to noise when the latter is clearly identified [30]. We also observed that the filter OT is not robust to image rotation when the images are noisy, especially if the noise cannot be explicitly evaluated, see Fig. 11 (different versions of this filter were considered in [30]).

The minimum average correlation energy (MACE) filter was also studied [11,33]. Its correlation performance is good when the target image is similar to one of the reference images, see the region B in Fig. 12. Nevertheless, the robustness of this filter is very poor since the correlation peak disappears in the $\mathrm{A}$ and $\mathrm{C}$ regions (Fig. 12). In [34], Iftekharuddin et al. proposed and validated an optimized MACE filter, the amplitude coupled MACE filter, that is robust to rotation. However, as the classical MACE filter the optimized version is also sensitive to noise, especially to structured noise.

Next, the AMPOF [16,35] was considered because it is high discriminating performance. Awwal et al. [35-36] suggested an optimization of the POF filter based on the following idea: the more the spectrum of the Fourier plane can be flattened the sharper will be the correlation peak. These authors introduced the amplitude-modulated phase-only filter $\left(H_{A M P O F}\right)$. As shown in our tests [30], the AMPOF is not robust to rotation and that is weakly robust to noise. However, the AMPOF is found to have significantly superior correlation discrimination capability. In [37], Iftekharuddin et al. studied a discretized version of the AMPOF filter. The resulting filter has good recognition performances of a nosiy object. In addition, the good discrimination performances of the AMPOF led Iftekharuddin et al. [38] to introduce an amplitude modulated inverse filter (AMIF) which is robust to Gaussian white noise. But, the AMIF is very sensitive to 
face rotation. Thus, we considered this AMPOF filter in order to compare it with our new ASPOF filter. This is to highlight the good discriminatory performance of our ASPOF filter. Another filter that was considered in this study is the OT-MACH filter [39-40]. From these different comparisons, the POF, AMPOF, and the MACH composite filters were selected for the comparison of correlation performances with our optimized ASPOF filter.

\subsection{Performance criterion adapted to the ASPOF}

Here, we will modify the definition of the PCE, i.e. Eq. (2), since we assumed that only a single correlation peak is considered, and that the filter which led to the correlation peak contains the total energy. These assumptions should be reconsidered for the ASPOF. Only half of the energy of the output plane is associated with each filter since the ASPOF is composed of two distinct filters. On the other hand, each filter leads to a correlation peak. Hence, for various situations two correlation peaks can appear in the output plane (see, e.g. Fig. 13 (a)). To circumvent the problem connected to the energy lowering of the correlation peak with the ASPOF, i.e. only half the filter plane is used, the correlation peak magnitude could be multiplied by a factor of 2 . However, this "trick" is insufficient. In fact the asymmetry feature of this filter leads to a correlation between the target image and a part of our filter, while there is intercorrelation with the second part. This can be observed in Fig. 13 which shows three correlation planes of the target image defined by the letter A.

As is apparent in Fig. 13, no correlation peaks are visible with the composite filters and AMPOF. However, it should be noted that the correlation plane displayed in Fig. 13 (a) shows two peaks: a first peak arises from the autocorrelation between the target image and the part of the ASPOF which contains the reference image which is similar to the target image, and the second peak can be identified as due to the intercorrelation between the target image and the 
other part of the ASPOF. Overall, the autocorrelation plane is noised by the intercorrelation plane. This effect has for effect to decrease the PCE values and consequently the identification performances of the ASPOF. In addition, it may happen that the target image is similar to two reference images, with one of them being in the first part of the ASPOF and the other one being in the second part of the ASPOF. To address this difficulty, we defined a different criterion, hereinafter denoted as the adapted peak-to-correlation energy (PCEA), which can be written as

$$
P C E A=\frac{\sum_{x=x_{0}-t} \sum_{y=y_{0}-t}^{x=x_{0}+t}|2 C(x, y)|^{2}}{\sum_{x=1}^{x=N} \sum_{y=1}^{y=M}|C(x, y)|^{2}+3\left(\sum_{x=x_{0}-t}^{x=x_{0}+t} \sum_{y=y_{0}-t}^{y=y_{0}+t}|C(x, y)|^{2}\right)}
$$

To further investigate this point, i.e. to separate the autocorrelation plane from the intercorrelation one, we have added a specific phase term to the two parts of the ASPOF. This phase is chosen that the correlation can be placed at a given point in the output plane. The respective correlation plane performances of the different simulations are in very good agreement with those gauged with the PCEA performance metric.

\section{Illustrating the ASPOF performances}

\subsection{Robustness against face rotation}

We considered the behavior of the 10-reference $H_{A S P O F}$ for the problem of identifying the letter A with a rotation angle ranging from $-20^{\circ}$ to $25^{\circ}$. Then, each letter $\mathrm{A}$ of the data-base (obtained by rotating the A image over $\left(-90^{\circ}, 90\right)$ in increments of $1^{\circ}$ counter clockwise) was correlated with the 10 -reference $H_{A S P O F}$. As shown by the green line in Fig. 14 (a), the letter A has been identified over $\left(-20^{\circ}, 25^{\circ}\right)$. To emphasize the benefit of the optimization stage concerning the isolated pixels (stage (iv) of the algorithm) on the filter behavior, it is instructive 
to contrast the results obtained for the $H_{A S P O F}$ without taking into account this step in our algorithm as shown by the green line in Fig. 14 (b). In the latter case, it should be noted that the PCE values are decreased by a factor of 2. It is evident that this optimization stage allows us to decrease the influence of the isolated pixels in the classical segmented filter $H_{S P O F}$.

For comparison, we also plot in Figs. 14 (a) and (b) the correlation performances obtained with $H_{P O F}^{1}$ (blue line), $H_{S P O F}$ (red line), and $H_{A M P O F}$ (black line) in the case of a noise free target image. Figs. 14 (a) and (b) show that $H_{A S P O F}$ is characterized by the largest PCE values in the correlation region $\mathrm{A}$, including those of the AMPOF. In addition, $H_{A S P O F}$ presents the most significant contrast between the correlation region $\mathrm{A}$ and the no-correlation regions $\mathrm{B}$ and $\mathrm{C}$. This shows that $H_{A S P O F}$ is robust in the correlation region and discriminating in the no-correlation regions.

\subsubsection{Robustness to rotation and noise}

As we next demonstrate, the conclusions are robust since ASPOF remains effective when noise is contained in the target image. The noise analysis procedure was identical to that

performed previously. Fig. 14 (c), corresponding to a centred white noise of variance set to 0.1 , and Fig. 14 (d) which considers a structured background noise. For each case, $H_{A S P O F}$ is a noise robust filter by comparison with the composite filters which were considered previously. Different tests involving other types of noise (not shown) are consistent with this observation.

\subsection{ROC curves}

The ROC curves in Fig. 15 demonstrate the effectiveness of the ASPOF for image recognition. The data base consisted of 10 reference images of the letter A (binary image, $512 \times 512$ pixels) taking a range for angle of rotation from $-60^{\circ}$ to $60^{\circ}$, i.e. $-55^{\circ},-45^{\circ},-30^{\circ},-15^{\circ}$, 
$0^{\circ}, 15^{\circ}, 30^{\circ}, 45^{\circ}, 55^{\circ}, 60^{\circ}$. The correlation planes are quantified using Eq. (2) and Eq. (5) (we choose $t=10$ pixels), and the recognition performance of composite filters for this specific set of reference images are studied and compared (supplemental material [30]). The merging of the two components of the ASPOF, i.e. $H_{A S P O F}=a H_{S P O F}^{1}+b H_{S P O F}^{2}$, was also optimized by choosing $a=2$ and $b=1.5$. Fig. 15 shows the ROC curves obtained with the capital letters A and V (by rotating the $\mathrm{V}$ letter in increments of $1^{\circ}$ counter clockwise from $-90^{\circ}$ to $90^{\circ}$ ) for different composite filters. As it can be seen in the ROC curves of Fig. 15 the AMPOF gives the closest results to a random guess. This reflects the strong discrimination and the low robustness to rotation of the AMPOF. From Fig. 15, we can see that the overall performance of the segmented filter is better than that of the AMPOF, but remains poor because of the isolated pixels. POF is able to achieve better performance than AMPOF; however due to the saturation problem only a weak difference between the good decisions and the false alarms is observed (Fig. 14). This is in contrast with the different performance points that can be achieved with the ASPOF. On one hand, the results of our tests clearly show the good recognition performance of the ASPOF, i.e. $\mathrm{TPR}=0.92$ corresponding to $\mathrm{FPR}=0$. On the other hand, it presents good separation between good decisions and false alarms (Fig. 14).

\subsection{Application to face recognition}

The final point we wish to mention here is that the above ideas can be extended to the problem of gray scale images identification. For this purpose we considered six different subjects from the PHPID [2]; an example is given in Fig. 16 (a) with several training images per person corresponding to different face orientations. As above, it is possible to define a 5-reference ASPOF for a given subject (with variable rotation angles, i.e. from top to bottom: $-15^{\circ}, 0^{\circ},+15^{\circ}$, 
and from left to right: $\left.+90^{\circ}, 0^{\circ},-90\right)$ in the following manner: 3 references were placed in the first half of the ASPOF and the remaining two references were positioned in the other half part of the ASPOF.

In this example, we choose $t=1$ pixel, $a=3$ and $b=1.5$. The values of these parameters are chosen in relation with the considered application. The 5 -reference images corresponds to $-45^{\circ}$,$30^{\circ},-15^{\circ}, 15^{\circ}, 45^{\circ}$ rotation angles (Fig. $16(\mathrm{~b})$ ). ASPOF training set correlations in Fig. 17 (a) were observed to produce good recognition performances in comparison to those of POF. It is likely that these differences originate from the saturation problem which is more pronounced for the case of faces. The recognition performance of the ASPOF was also compared to that of the optimal trade-off MACH (OT MACH) filter with a background noise. The parameters for the OT MACH are $\alpha=0,25, \beta=1$, and $\gamma=0,1[30,39-40]$. The results in Fig. 17 (b) show that the recognition performances are significantly larger with the ASPOF. The result (Fig. 17 (b)) of our method demonstrates the good recognition performance of the ASPOF, i.e. TPR $=0.69$ corresponding to $\mathrm{FPR}=0$ to be compared with $\mathrm{TPR}=0.33$ and $\mathrm{TPR}=0.47$ for the $\mathrm{OT} \mathrm{MACH}$ and POF, respectively).

\section{Summary}

In summary, we have developed a face recognition system based on optical correlation for use in identification and classification. The system will be able to detect, identify and track various targets with robustness and discrimination. The method has two main stages: increase the

number of reference images in order to consider as much as possible target image changes, and use composite filters. The main advantage of these filters is to merge the information of many reference images in a single filter, thus reducing the number of correlations to make a reliable 
decision. However, the robustness and discrimination performances decrease as the number of reference images is increased, i.e. saturation. To overcome this limitations, we have presented herein a fairly thorough analysis of an optimized composite filter, i.e. the asymmetric segmented phase only filter (ASPOF), for improving the effectiveness of a VLC when used for face identification. The principle of our filter consists in a specific segmentation of the filter's Fourier plane in several regions. Next, each region was assigned to a single reference image. This optimization stage allowed us to increase significantly the number of reference images of the segmented filter. However, the main drawback of this solution is to generate isolated pixels which yield to low overall performances of this kind of filter. Next, we have proposed a new type of optimized composite filter, i.e. the ASPOF. We found useful to introduce a new segmentation criterion to characterize the correlation plane of this filter. Each of the stages has various parameters that must be optimized in order to increase the robustness and discriminating ability while increasing the number of reference images contained in the filter. We have validated this technique and applied it to several binary and gray scale images identification. Overall, our results are encouraging and demonstrate that the ASPOF is characterized by a good compromise between robustness and discrimination.

Furthermore, we believe that the simplicity of the technique provides sufficient appeal from the experimental viewpoint. Of equal or potentially more importance will be to optically or numerically implement it. We believe that it would be interesting to explore this topic further-we discuss very briefly below how this might be done. There are two key directions for improving the results presented here. First, a careful optimization of the merging method is required to maximize the use of the filter's Fourier plane bandwidth. Second, we expect that 
these general results will apply in 3D face recognition application as well as well. Work addressing these avenues has been initiated.

\section{Acknowledgments}

The authors acknowledge the partial support of the Conseil Régional de Bretagne and thank A. Arnold-Bos (Thales Underwater Systems) for helpful discussions. They also acknowledge S. Quasmi for her help with the simulations. Lab-STICC is Unité Mixte de Recherche CNRS 3192. 


\section{References}

1. A. VanderLugt, "Signal detection by complex spatial filtering," IEEE Trans. Inf. Theor. 10, 139-145 (1964).

2. Pointing Head Pose Image http://www.ecse.rpi.edu/ cvrl/database/other_Face_Databases.htm

3. C. S. Weaver and J. W. Goodman, "A technique for optically convolving two functions," Appl. Opt. 5, 1248-1249 (1966).

4. A. Alfalou and C. Brosseau "Understanding Correlation Techniques for Face Recognition: From Basics to Applications," in Face Recognition, Milos Oravec (Ed.), ISBN: 978-953307-060-5, In-Tech (2010).

5. J. L. Horner and P. D. Gianino, "Phase-only matched filtering," Appl. Opt. 23, 812-816 (1984).

6. F. T. S. Yu, S. Jutamulia "Optical Pattern Recognition," Cambridge University Press (1998).

7. B. V. K. Vijaya Kumar, "Tutorial survey of composite filter designs for optical correlators," Appl. Opt. 31, 4773-4801 (1992).

8. A. Alfalou, G. Keryer, and J. L. de Bougrenet de la Tocnaye, "Optical implementation of segmented composite filtering," Appl. Opt. 38, 6129-6135 (1999).

9. H. J. Caufield and W. T. Maloney, "Improved discrimination in optical character recognition,” Appl. Opt. 8, 2354-2356 (1969).

10. C. F. Hester and D. Casasent, "Multivariant technique for multiclass pattern recognition," Appl. Opt. 19, 1758-1761 (1980). 
11. A. Mahalanobis, B. V. K. Vijaya Kumar, and D. Casassent, "Minimum average correlation energy filters,” Appl. Opt. 26, 3633-3640 (1987).

12. A. Alfalou and C. Brosseau, "Robust and discriminating method for face recognition based on correlation technique and independent component analysis model," Opt. Lett. 36, 645647 (2011).

13. M. Elbouz, A. Alfalou, and C. Brosseau, "Fuzzy logic and optical correlation-based face recognition method for patient monitoring application in home video surveillance," Opt. Eng. 50, 067003(1)-067003(13) (2011).

14. I. Leonard, A. Arnold-Bos, and A. Alfalou, "Interest of correlation-based automatic target recognition in underwater optical images: theoretical justification and first results," Proc. SPIE 7678, 767800 (2010).

15. V. H. Diaz-Ramirez, "Constrained composite filter for intraclass distortion invariant object recognition ," Opt. Lasers Eng. 48, 1153 (2010).

16. A. A. S. Awwal, "What can we learn from the shape of a correlation peak for position estimation?," Appl. Opt. 49, B40-B50 (2010).

17. A. Alsamman and M. S. Alam, "Comparative study of face recognition techniques that use joint transform correlation and principal component analysis," Appl. Opt. 44, 688-692 (2005).

18. S. Romdhani, J. Ho, T. Vetter, and D. J. Kriegman, "Face recognition using 3-D models: pose and illumination," in Proceedings of the IEEE 94, 1977-1999 (2006).

19. J. L. Tribillon, “Corrélation Optique,” Edition Teknéa, Toulouse, (1999).

20. B. Javidi and C.- J. Kuo, "Joint transform image correlation using a binary spatial light modulator at the Fourier plane,” Appl. Opt. 27, 663-665 (1988). 
21. B. Javidi, "Nonlinear joint power spectrum based optical correlation," Appl. Opt. 28, 23582367 (1989).

22. M. S. Alam and M. A. Karim, "Fringe-adjusted joint transform correlation," Appl. Opt. 32, 4344-4350 (1993).

23. M. S. Alam and S. F. Goh, "Three-dimensional fringe-adjusted joint transform correlation," Appl. Opt. 43, 3680-3685 (2004).

24. G. Keryer, J. L. de Bougrenet de la Tocnaye, and A. Alfalou, "Performance comparison of ferroelectric liquid-crystal-technology-based coherent optical multichannel correlators," Appl. Opt. 36, 3043-3055 (1997).

25. A. Alfalou, "Implementation of Optical Multichannel Correlators: Application to Pattern Recognition,” PhD Thesis, Université de Rennes 1-ENST Bretagne, Rennes-France (1999).

26. B. V. K. Vijaya Kumar and L. Hassebrook, "Performance measures for correlation filters," Appl. Opt. 29, 2997-3006 (1990).

27. J. L. Horner, "Metrics for assessing pattern-recognition performance," Appl. Opt. 31, 165166 (1992).

28. J. P. Egan, Signal Detection Theory and ROC Analysis (Academic Press Series in Cognition and Perception, New York, 1975).

29. A. Pe'er, D. Wang, A. W. Lohmann, and A. A. Friesem, "Apochromatic optical correlation," Opt. Lett. 25, 776-778 (2000).

30. See supplemental material at http:// ... for further technical details and data analysis

31. M. Rahman, A. A. S. Awwal and K. S. Gudmundsson, "Composite filter for search time reduction for 3D model based object recognition", Proc. of SPIE 5201, 97-107 (2003). 
32. T. Mohammad, M. Rahman, A. A. S. Awwal, and K. S. Gudmundsson, "Optical pattern recognition of three dimensional images using composite binary phase only filters", Proc. of SPIE 5556, 146-157 (2004).

33. D. Casasent and G. Ravichandran, "Advanced distorsion-invariant MACE filters," Appl. Opt. 31, 1109-1116 (1992).

34. K. M. Iftekharuddin, F. Ahmad, and M. A. Karim, "Rotation invariant target recognition using amplitude coupled minimum average correlation energy filter", Opt. Eng. 35, 10091014 (1996).

35. A. A. S. Awwal, M. A. Karim, and S.R. Jahan, "Improved correlation discrimination using an amplitude-modulated phase-only filter," Appl. Opt. 29, 233-236 (1990).

36. A. A. S. Awwal, W. A. McClay, W. S. Fergusson, J. V. Candy, J. T. Salmon, and P. J. Wegner, "Detection and tracking of the back reflection of KDP images in the presence or absence of a phase mask", Appl. Opt. 45, 3038-3048 (2006).

37. K. M. Iftekharuddin, M. A. Karim, P. W. Eloe, and A. A. S. Awwal, "Discretized amplitude modulated phase only filter", Opt. Laser Technol. 28, 93-100 (1996).

38. K. M. Iftekharuddin, M. A. Karim, and A. A. S. Awwal, "Optimization of amplitude modulated inverse filter", Math. Comput. Model. 24, 103-112 (1996).

39. A. Mahalanobis, B. K. V. Vijaya Kumar, S. Song, S. R. F. Sims, and J. F. Epperson, “Unconstrained correlation filters," Appl. Opt. 33, 3751-3759 (1994).

40. H. Zhou and T.- H. Chao, "MACH filter synthesizing for detecting targets in cluttered environment for grayscale optical correlator,” Proc. SPIE 3715, 394 (1999). 


\section{Table}

Table 1: Illustrating the different composite filters used in this study. $H_{{ }_{c o m p}{ }_{A d}}$ denotes the adapted composite filter. It is realized by considering a linear combination of reference images and using the adapted filter definition. $H_{\text {Comp-POF }}$ is the POF composite filter. We tested two different schemes for realizing the composite POF filter. In the first scheme $\left(H_{c o m p}^{1}\right.$ PoF $)$, a linear combination of reference images was used to create the POF. The second scheme $\left(H_{c o m p}^{2}{ }_{P O F}\right)$ involves performing the POFcfor each reference, and using the linear combination of these

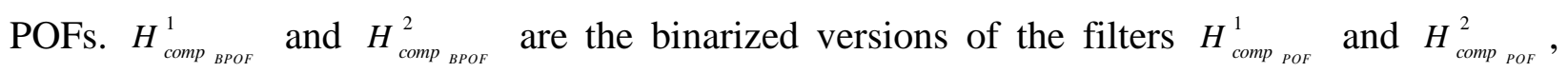
,respectively. The composite inverse filter $H_{\text {comp }{ }_{I F}}$ is the inverse filter of the linear combination of reference images. The optimal composite filter $H_{\text {соmp } p_{\text {o }}}$ is realized by linearly combining reference images. $H_{\text {comp spof }}^{1}$ denotes the segmented filter realized by doing segmentation and assignment with the energy criterion. The calculation of filter $H_{\text {comp sPoF }}^{2}$ is done by replacing the energy with the square of the real part of the different references spectra to be merged. $H_{\text {comp }_{\text {MACE }}}$ is the composite filter of the MACE filter. $H_{\text {comp }}^{\text {AMPOF }}$ is the composite version of the AMPOF. 


\section{Figure Captions}

FIG. 1: Schematic of the principle of the VLC scheme.

FIG. 2: Illustrating the saturation effect: (a) three 8-bit grey scale images. (b) Image obtained by a classical linear combination of the three images shown in (a). (c) Image obtained using an optimized merging (spectral segmentation).

FIG. 3: Technique used to classify the reference images in 2 sub-classes.

FIG. 4: Illustrating the optimized assignment procedure for isolated pixels.

FIG. 5: Merging technique based on the Fourier plane symmetry property.

FIG. 6: (Color online) PCEs obtained with 10-reference POF, SPOF, AMPOF, and ASPOF. (a) $t=1$, (b) $t=5$, (c) $t=10$, and (d) $t=20$.

FIG. 7: (Color online) PCEs obtained with a 10-reference ASPOF and $t=10$ pixels. (a) $p_{1}=p_{2}=1$ (b) $p_{1}=2$ and $p_{2}=1.5$.

FIG. 8: (Color online) PCEs obtained with the composite POF. The colors shown in the inset denote the different filters as a function of the number of references used. 
FIG. 9: (Color online) Discrimination results: (a) the target image is the letter V. (b) PCEs obtained with a filter fabricated with reference images of letter A. The colors shown in the inset denote the different filters as a function of the number of references used.

FIG. 10: (Color online) PCEs obtained with the inverse composite filter. The colors shown in the inset denote the different filters against the number of references used.

FIG. 11: (Color online) (a) Illustrating the letter A with additive background noise. (b) Same as in (a) with a rotation angle of $-50^{\circ}$. (c) Illustrating the letter A with structured noise. (d) Same as in (c) with a rotation angle of $50^{\circ}$. (e) Illustrating the letter A for a weak contrast. (f) PCEs obtained with the OT composite filter taking $\alpha=0.6$. The colors shown in the inset denote the different filters versus the number of references used.

FIG. 12: (Color online) PCEs obtained with a 10-reference MACE when the target images are noiseless. Several examples of the rotated letter A are shown at the bottom of this figure. The inset shows two correlation planes. On the right hand: autocorrelation obtained without rotation,. On the left hand, inter-correlation obtained with the letter A oriented at $-75^{\circ}$.

FIG. 13: Output normalized Fourier planes obtained by correlating of the same target image (letter A with a rotation angle of $-75^{\circ}$ ) with: (a) ASPOF, (b) composite filter, and (c) AMPOF.

FIG. 14: (Color online) Comparison between the different correlations of letter A (we consider rotation angles ranging between $-90^{\circ}$ and $90^{\circ}$ ) with the 10-reference composite filters: POF (blue 
line), Segmented (red line), AMPOF (black line), and ASPOF (green line). (a) PCEs obtained using the optimization stage concerning the isolated pixels, (b) PCEs obtained without the optimization stage concerning the isolated pixels. (c) and (d) represent the PCEs obtained with noised target images.

FIG. 15: (Color online) ROC curves obtained with 10-reference composite filters: POF (red), SPOF (green), AMPOF (purple) and ASPOF (navy blue). The sky-blue line shows the random guess.

FIG. 16: Example of face images from PHPID, that were captured under variable angle conditions, i.e. from top to bottom: $+15^{\circ}, 0^{\circ},+15^{\circ}$, and from left to right: $-90^{\circ}, 0^{\circ},+90^{\circ}$ : (a) target faces, (b) references.

FIG. 17: (Color online) (a) ROC curves obtained by correlating faces of a given subject, e. g. Fig. 9 (a), with 6 other individuals with 5-reference ASPOF (navy blue) and POF (red) composite filters. The sky-blue line shows the random guess. (b) ROC curve obtained with an OT MACH. 


\begin{tabular}{|c|c|}
\hline Composite filter & Notation \\
\hline Adapted filter & $H_{\text {comp }_{A d}}$ \\
\hline Phase-only filter & $H_{\text {comp }_{P O F}}^{1}, H_{\text {comp }_{P O F}}^{2}$ \\
\hline $\begin{array}{l}\text { Binary phase- } \\
\text { only filter }\end{array}$ & $H_{\text {comp }_{B P O F}}^{1}, H_{\text {comp }_{B P O F}}^{2}$ \\
\hline Inverse filter & $H_{\text {comp }_{I F}}$ \\
\hline $\begin{array}{l}\text { Compromise } \\
\text { optimal filter }\end{array}$ & $H_{\text {comp }_{\text {oT }}}$ \\
\hline Segmented filter & $H_{S P O F}^{1}, H_{S P O F}^{2}$ \\
\hline $\begin{array}{l}\text { Segmented binary } \\
\text { filter }\end{array}$ & $H_{B S P O F}^{1}, H_{B S P O F}^{2}$ \\
\hline $\begin{array}{l}\text { Minimum } \\
\text { average } \\
\text { correlation } \\
\text { energy filter }\end{array}$ & $\overline{H_{\text {comp }_{\text {MACE }}}}$ \\
\hline $\begin{array}{c}\text { Amplitude } \\
\text { modulated phase- } \\
\text { only filter }\end{array}$ & $H_{\text {comp }_{\text {AMPOF }}}$ \\
\hline
\end{tabular}

Table. 1: Leonard, Alfalou and Brosseau 


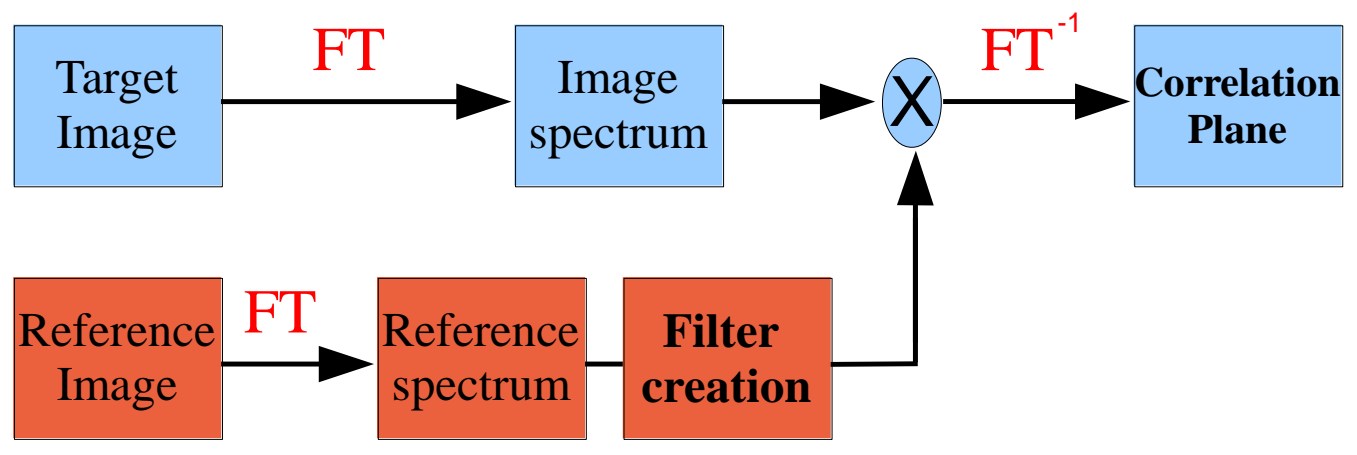

FIG. 1: Leonard, Alfalou and Brosseau 


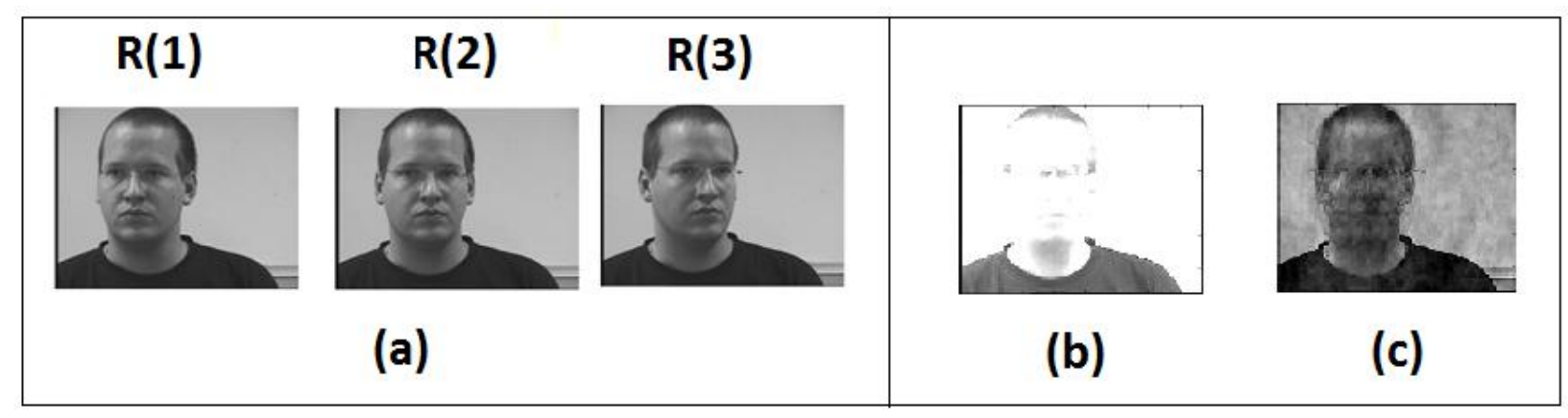

FIG. 2: Leonard, Alfalou and Brosseau 


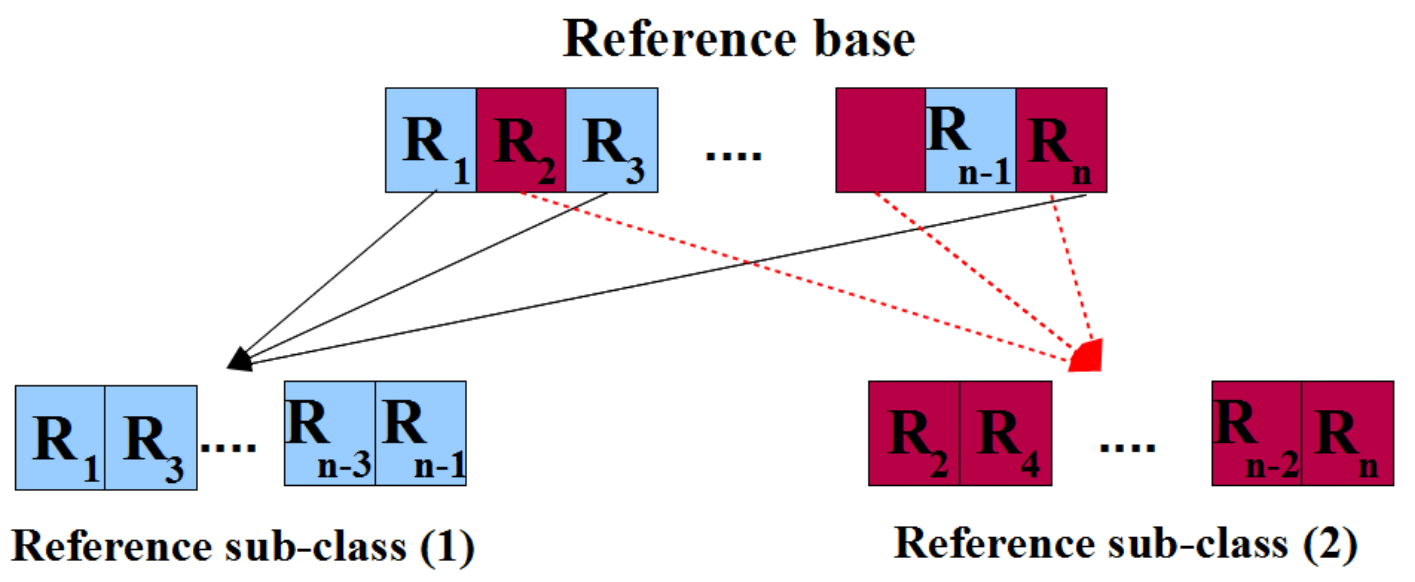

FIG. 3: Leonard, Alfalou and Brosseau 


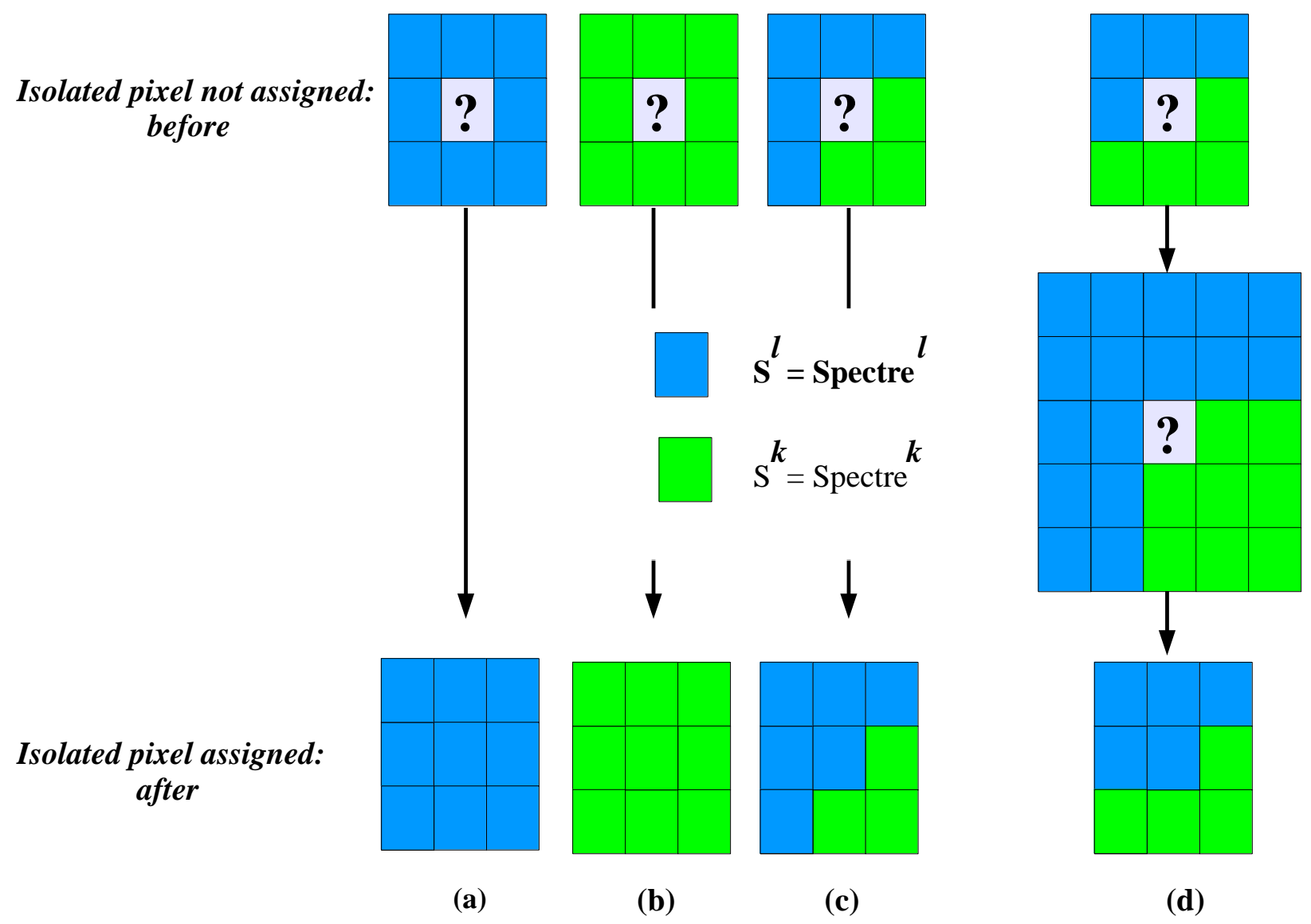

FIG. 4: Leonard, Alfalou and Brosseau 


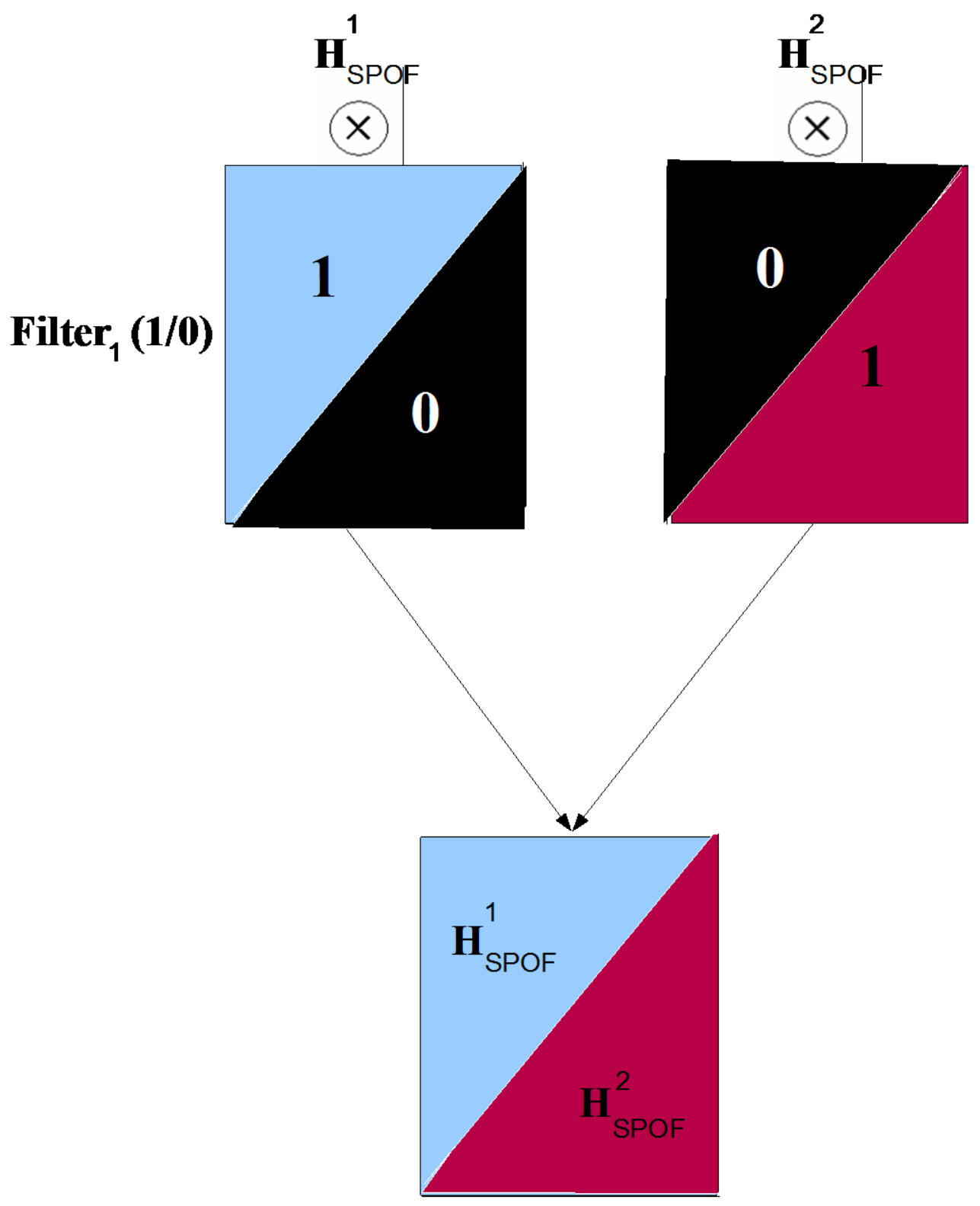

Filter $_{2}(\mathbf{0} / \mathbf{1})$

Asymmetric Segmented Phase Only Filter

FIG. 5: Leonard, Alfalou and Brosseau 


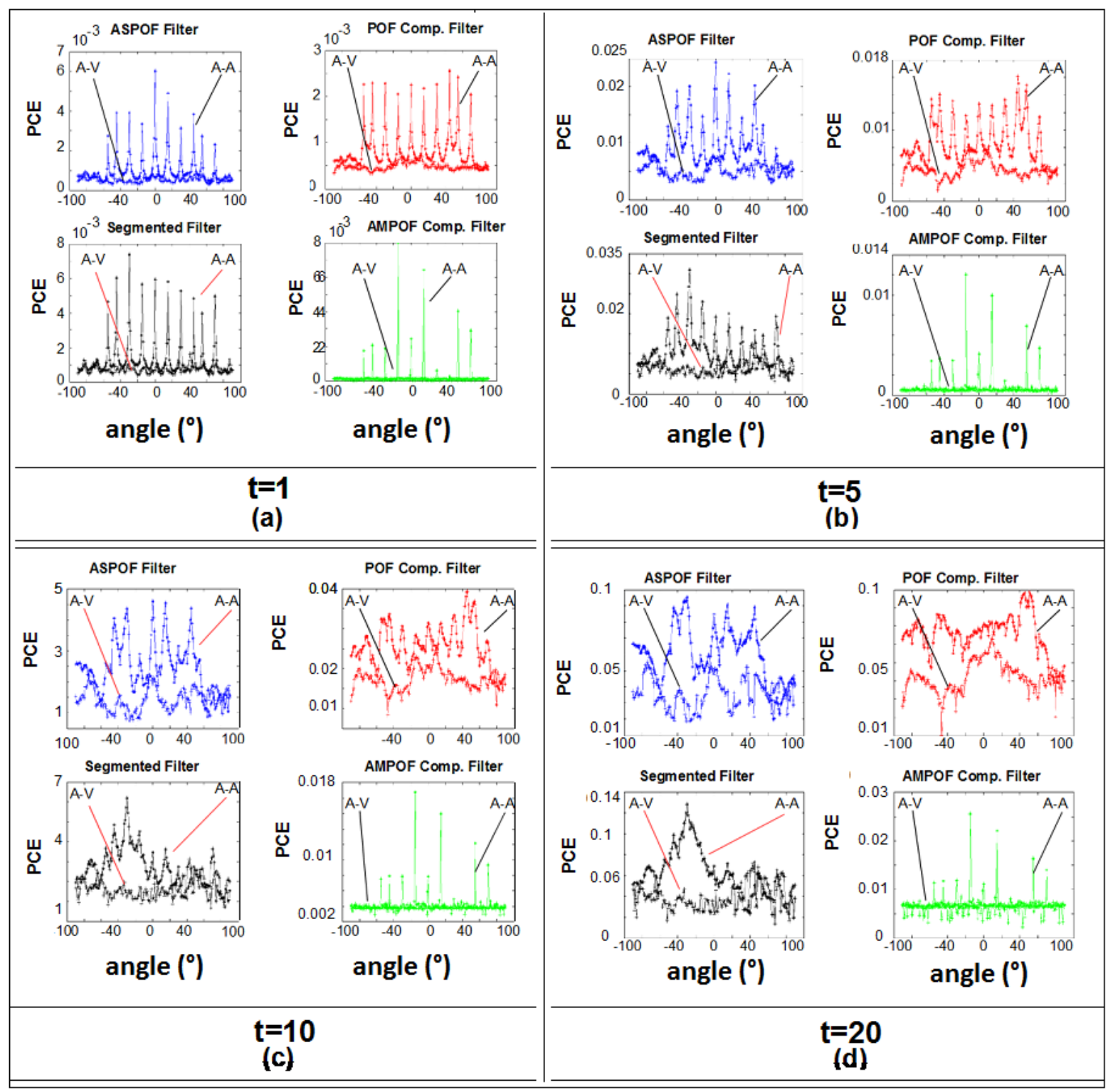

FIG. 6: Leonard, Alfalou and Brosseau 


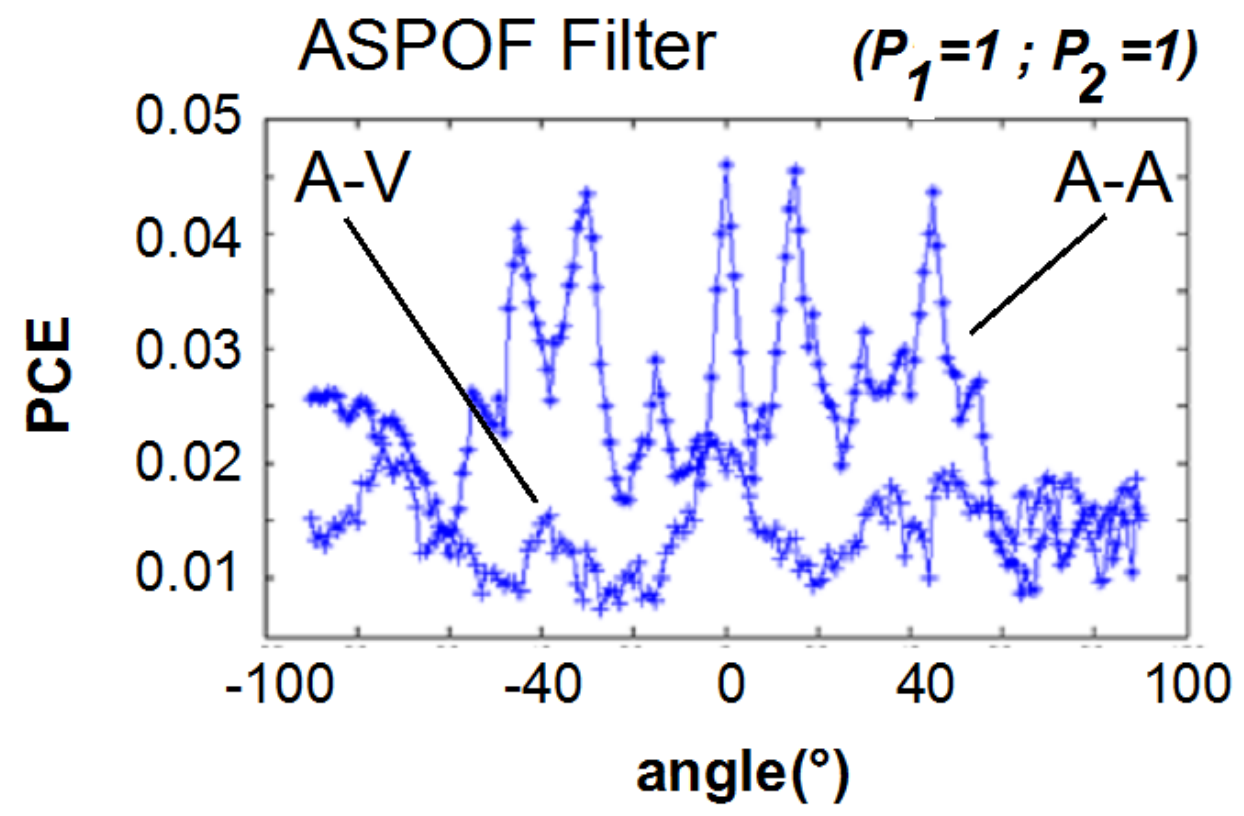

(a)

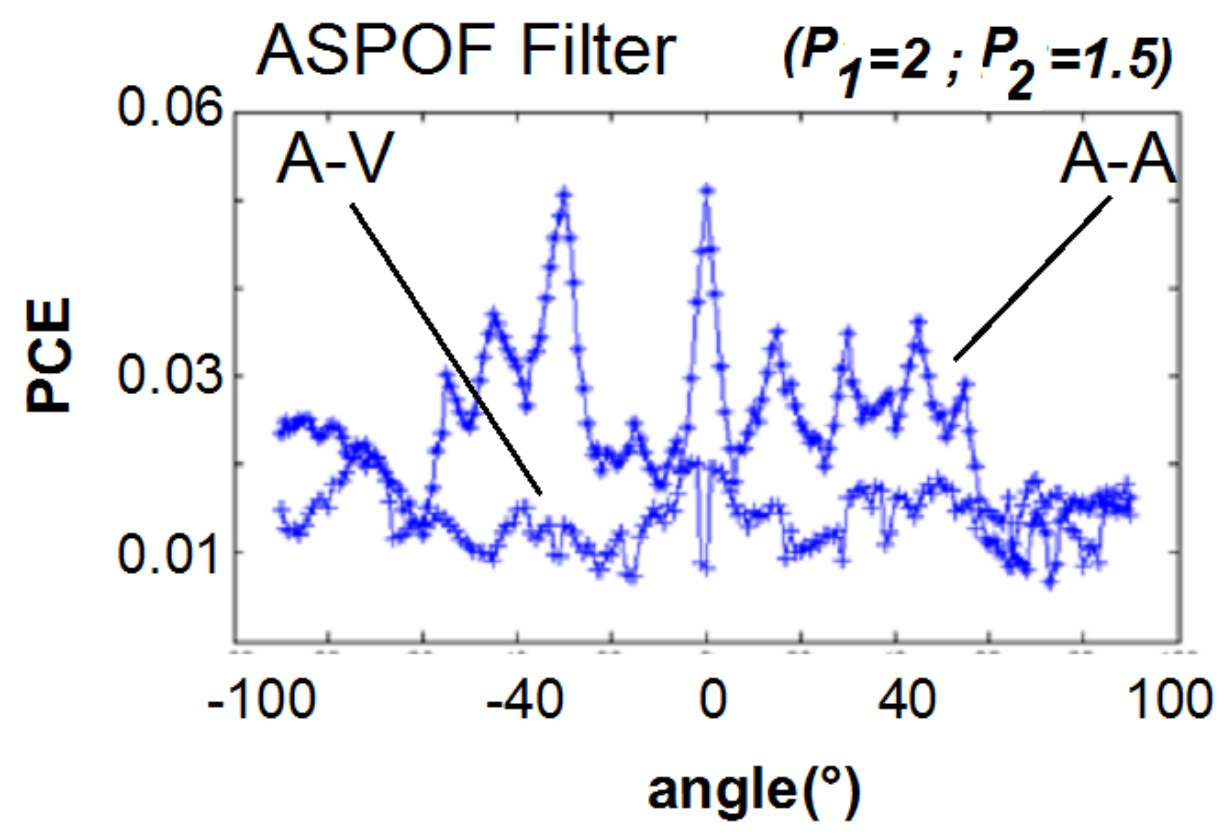

(b)

FIG. 7: Leonard, Alfalou and Brosseau 


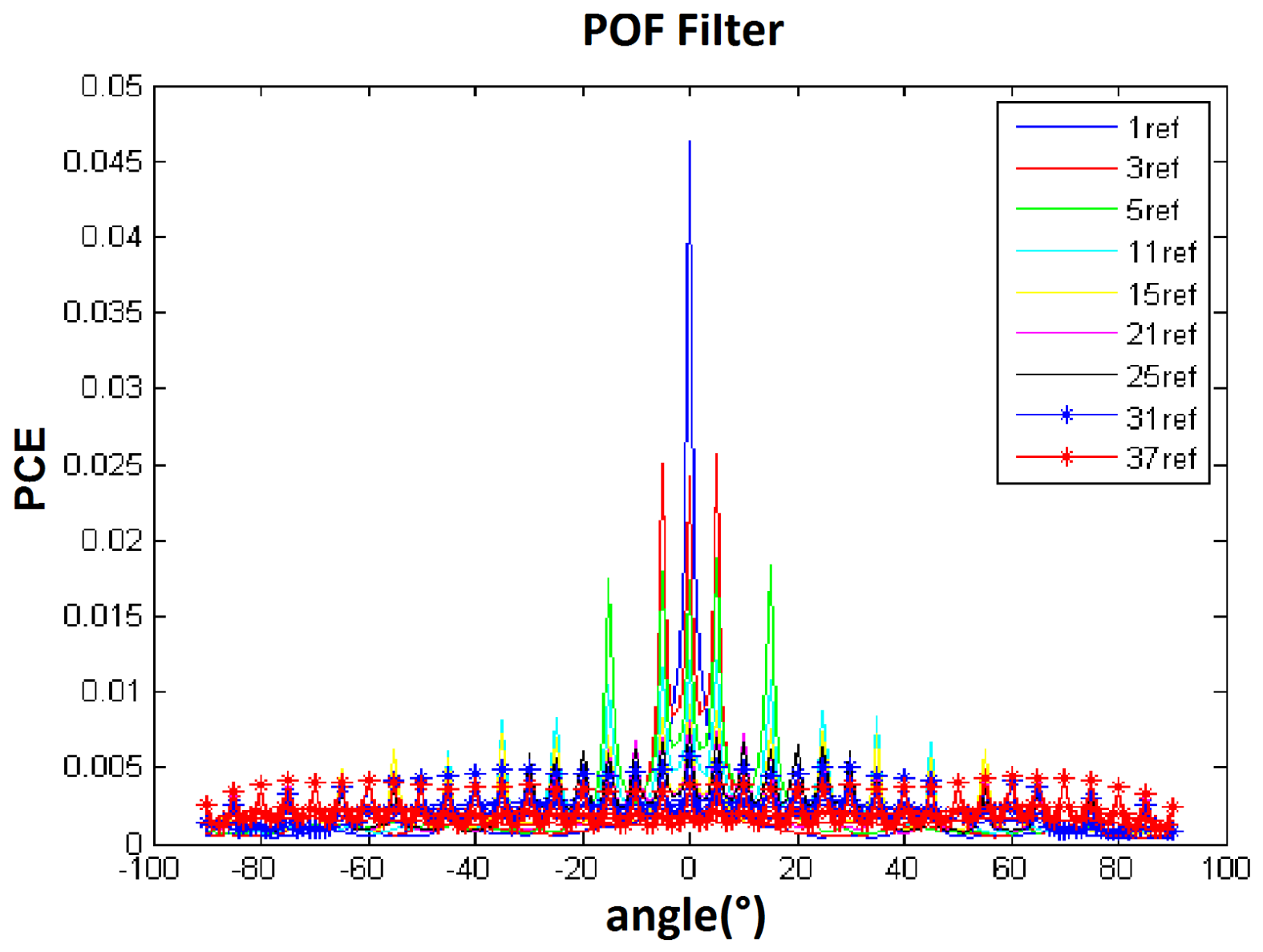

FIG. 8: Leonard, Alfalou and Brosseau 


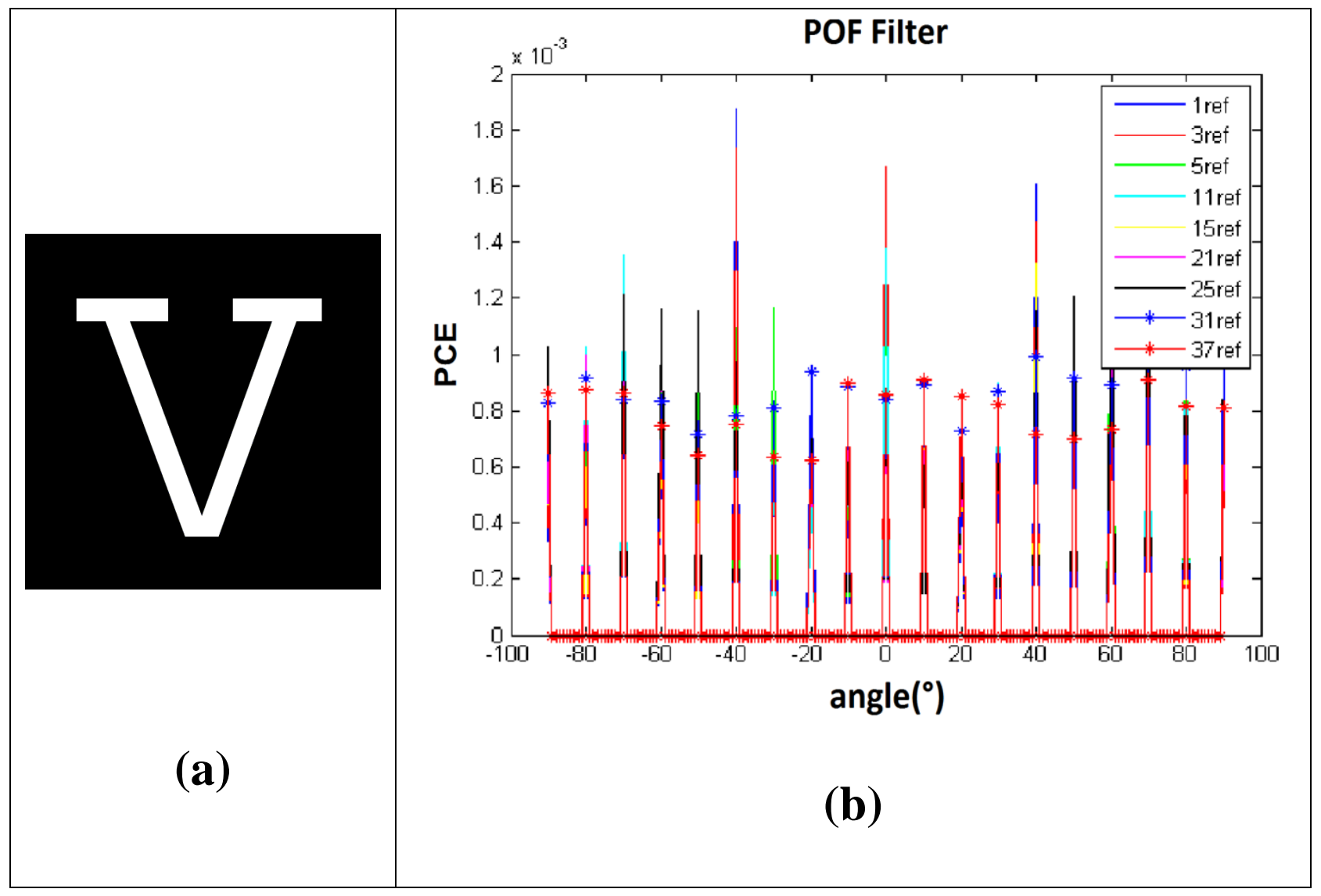

FIG. 9: Leonard, Alfalou and Brosseau 


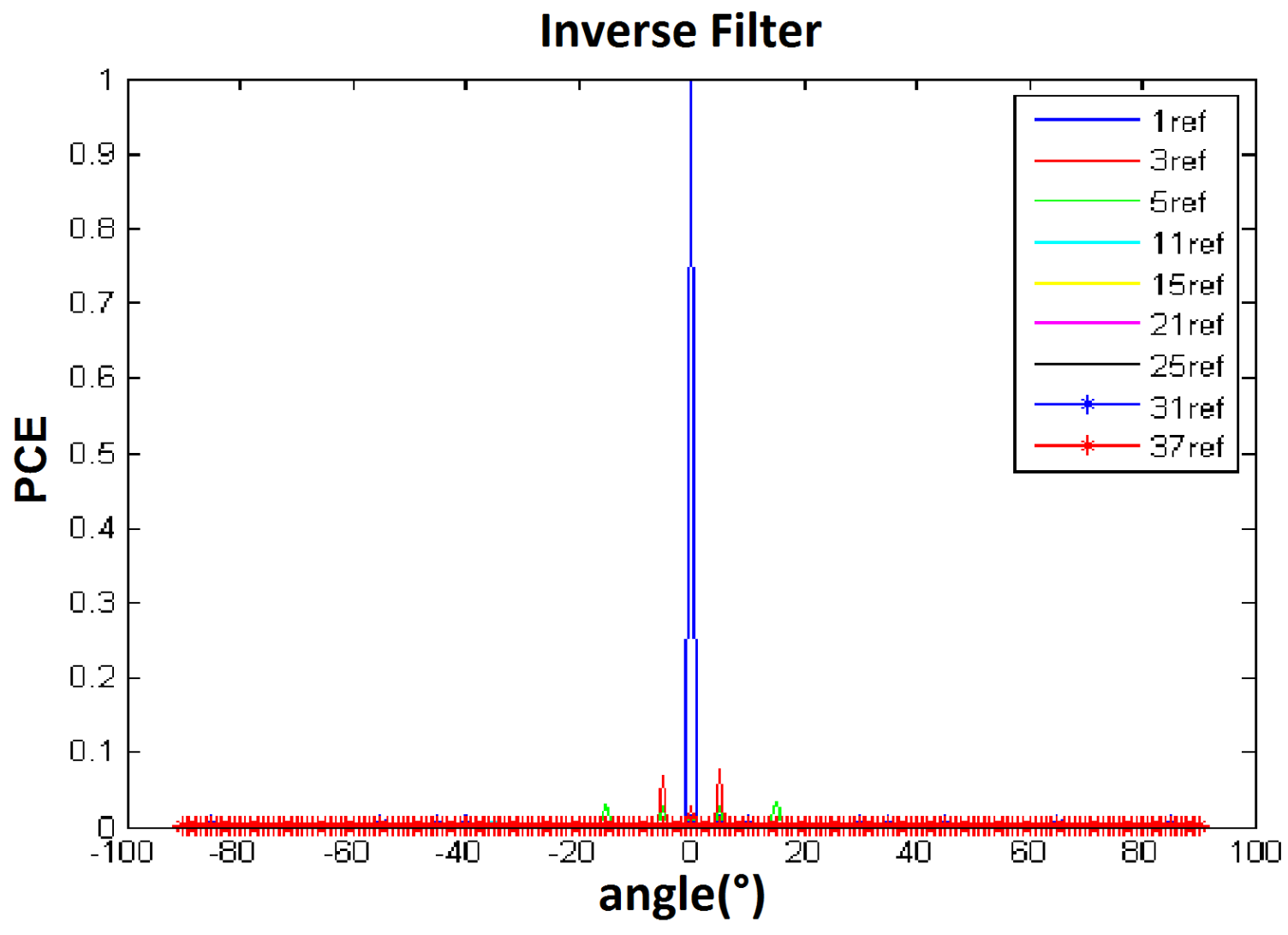

FIG. 10: Leonard, Alfalou and Brosseau 


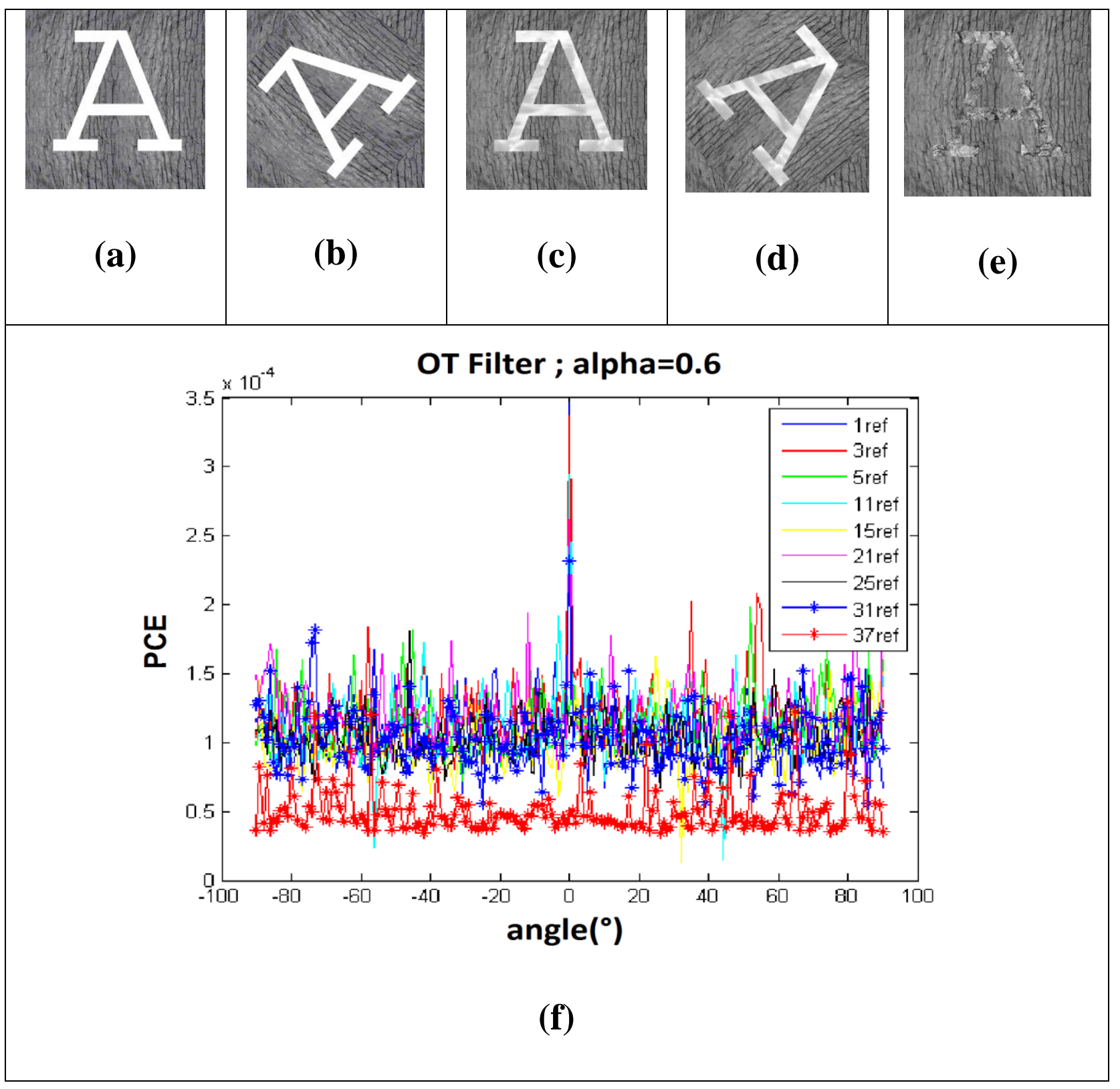

FIG. 11: Leonard, Alfalou and Brosseau 


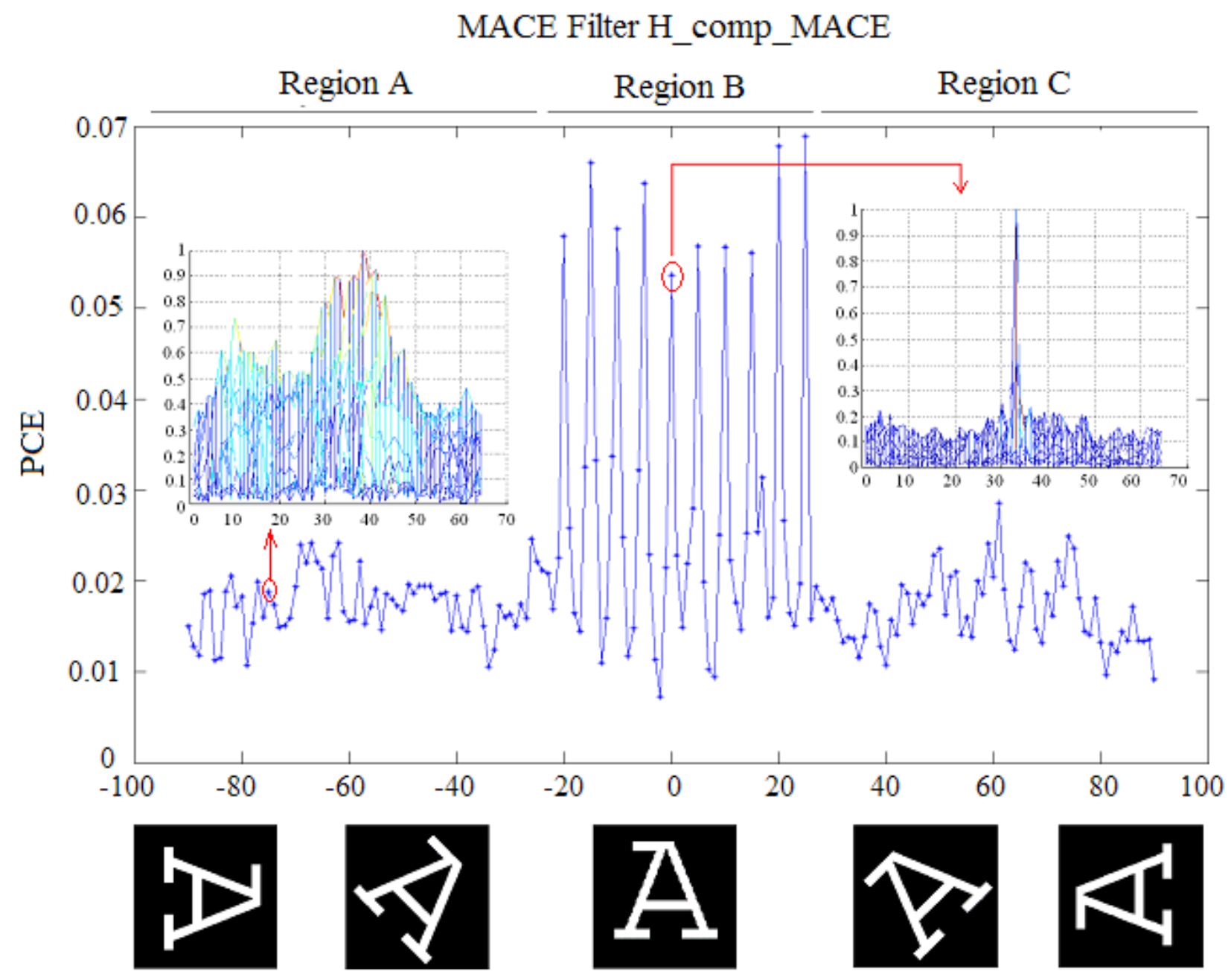

FIG. 12: Leonard, Alfalou and Brosseau 


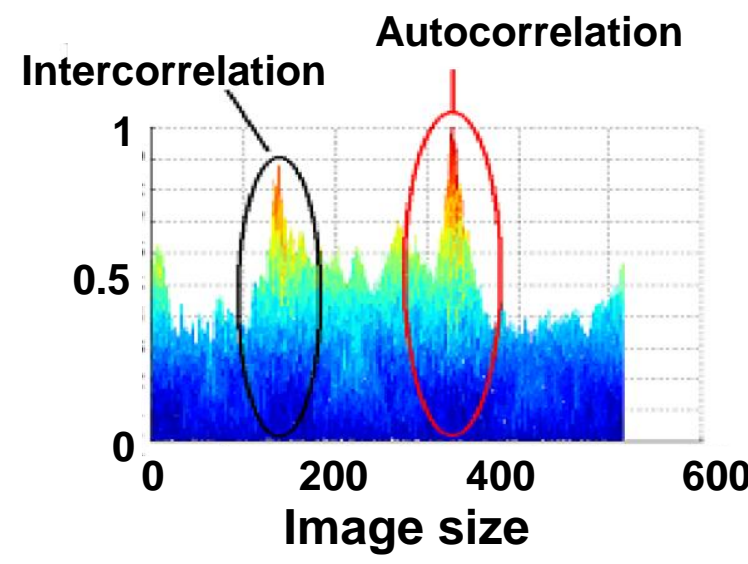

\section{ASPOF}

(a)

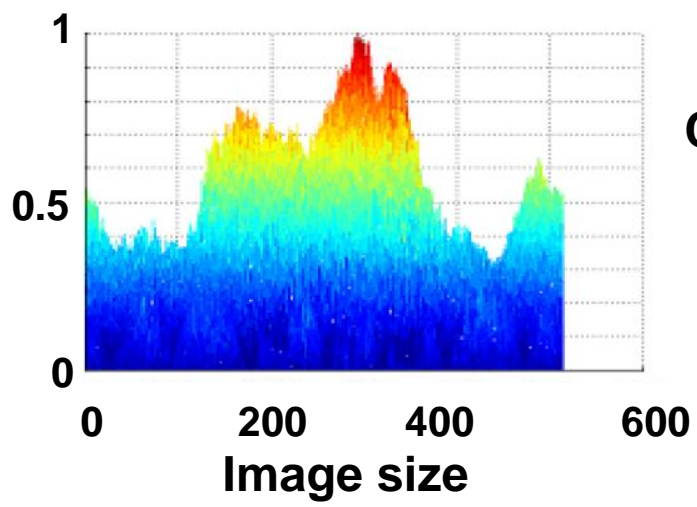

Composite

(b)

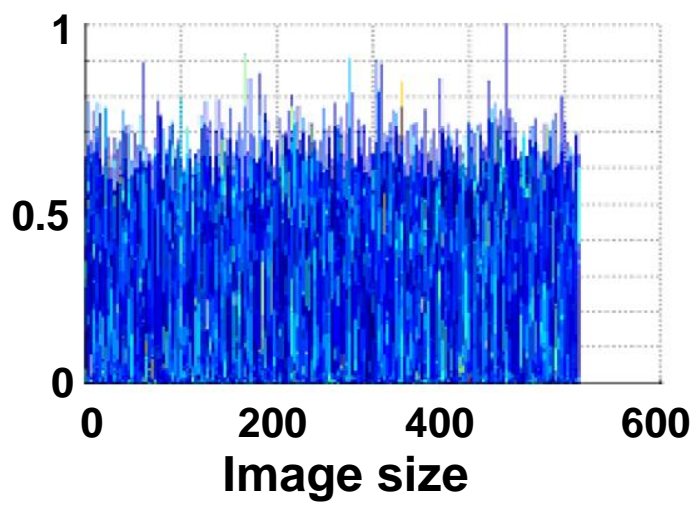

AMPOF

(c)

FIG. 13: Leonard, Alfalou and Brosseau 


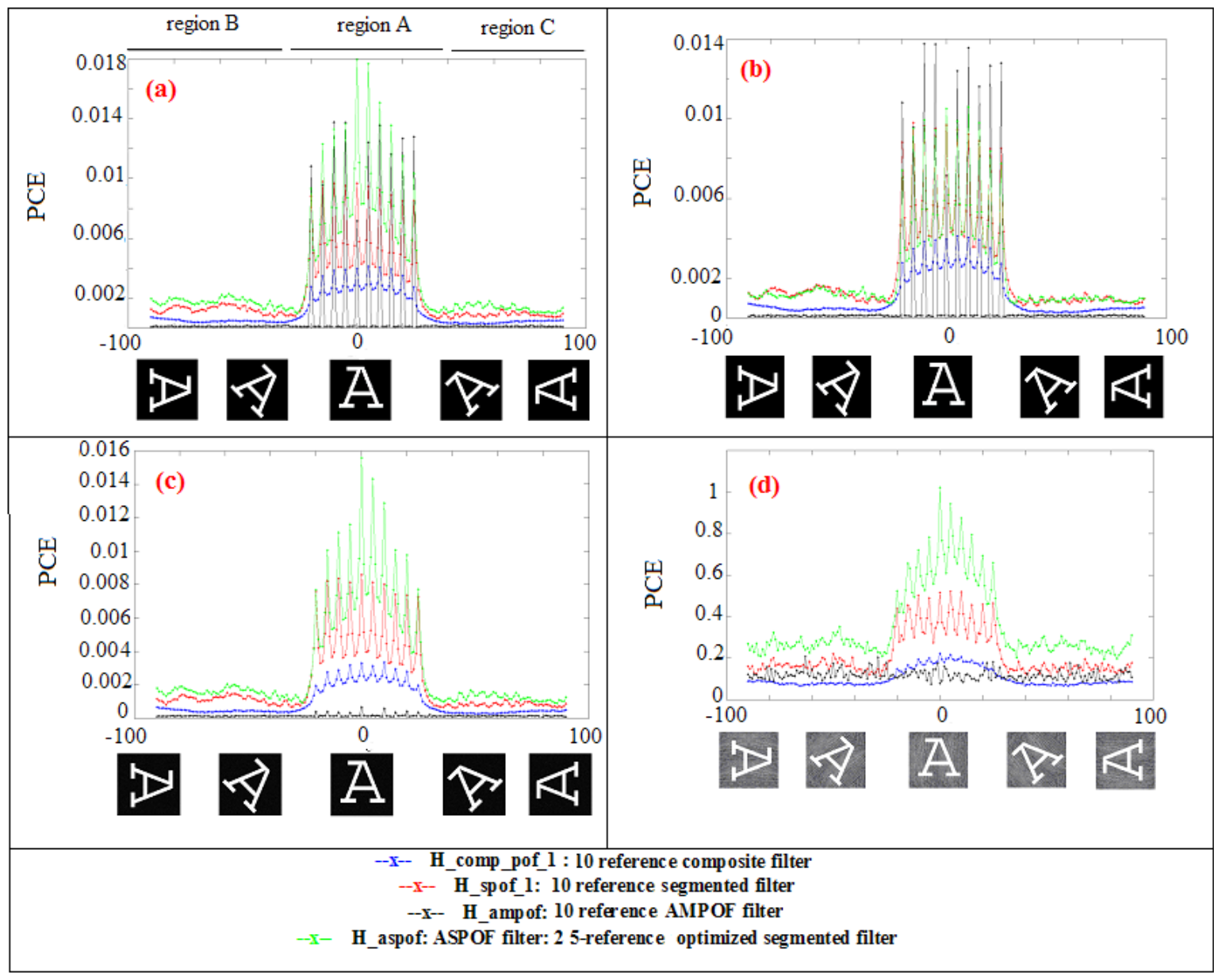

FIG. 14: Leonard, Alfalou and Brosseau 


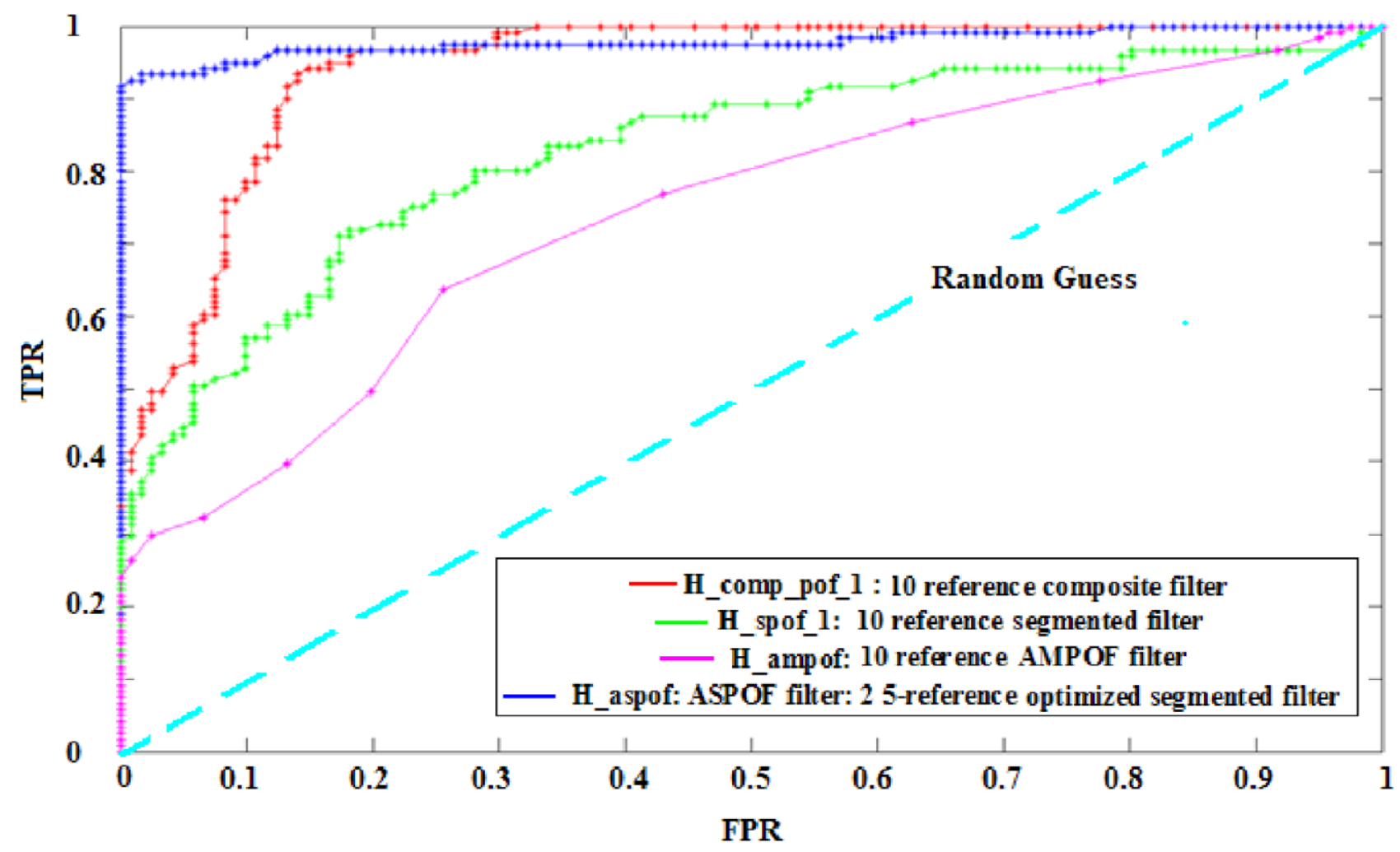

FIG. 15: Leonard, Alfalou and Brosseau 


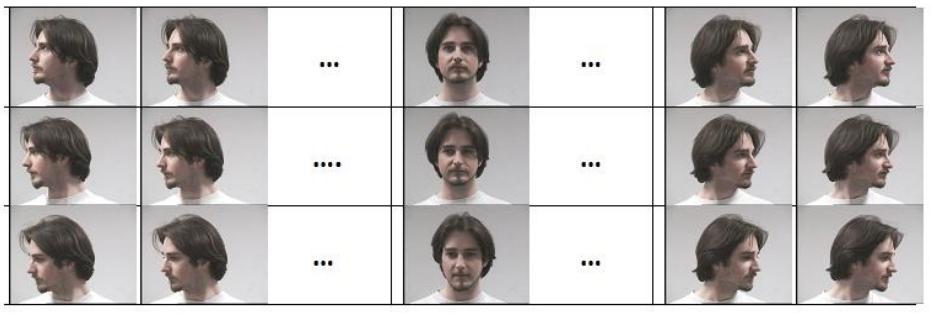

(a)
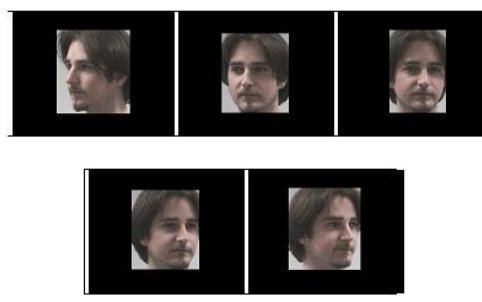

(b)

FIG. 16: Leonard, Alfalou and Brosseau 


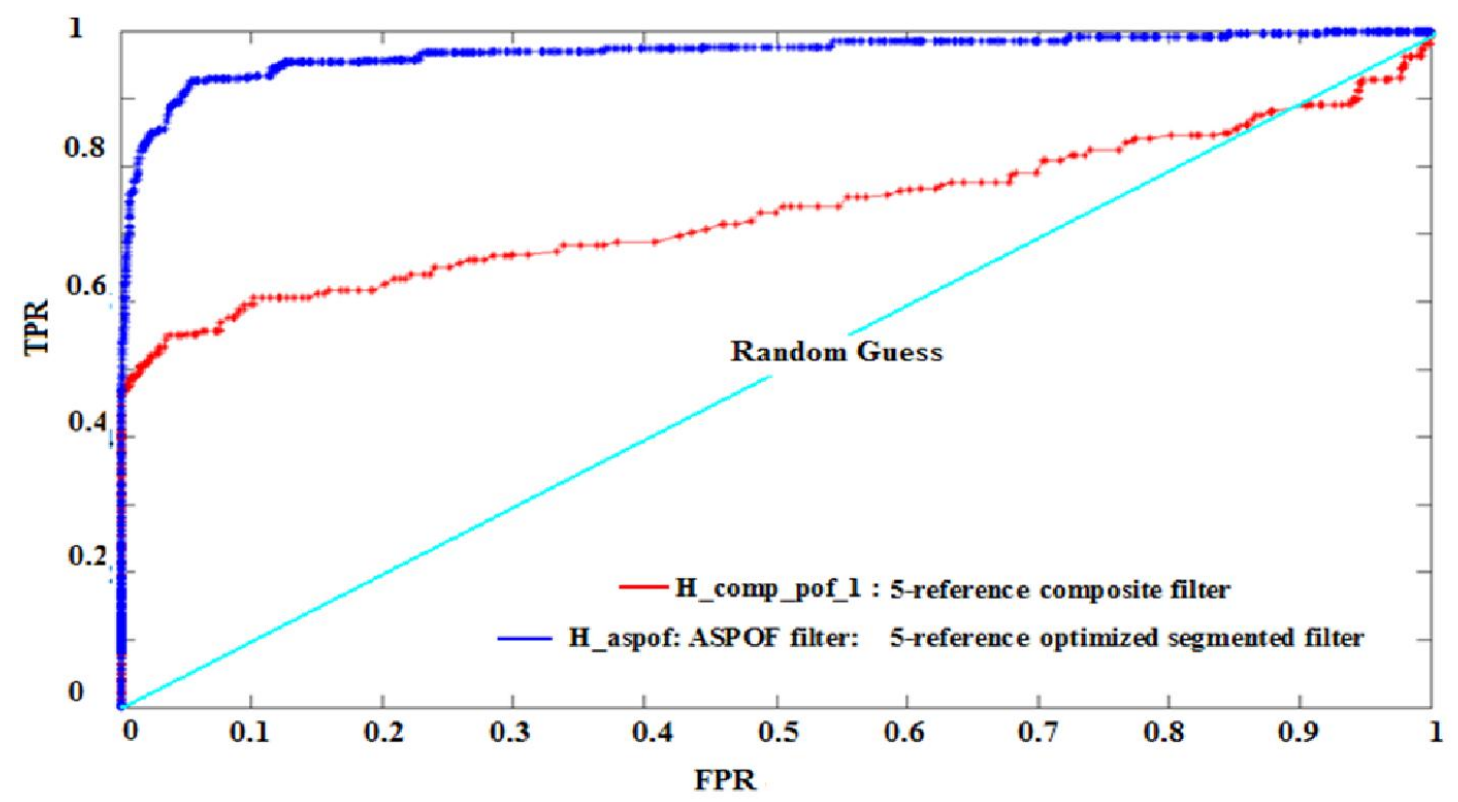

(a)

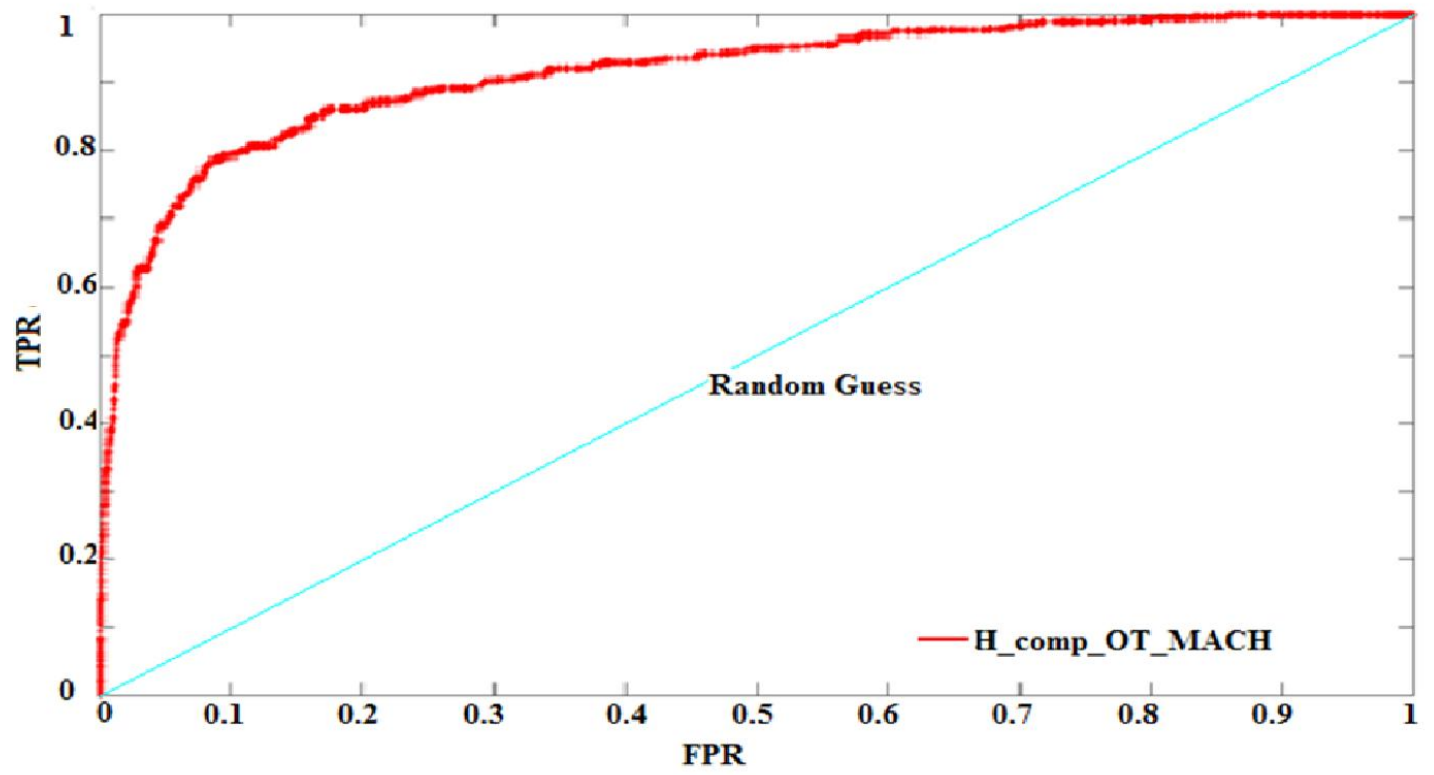

(b)

FIG. 17: Leonard, Alfalou and Brosseau 